\title{
Majorana anyon composites in magneto-photoluminescence spectra of natural quantum Hall puddles.
}

\author{
Alexander. M. Mintairov ${ }^{1,2, *}$, Dmitrii. V. Lebedev ${ }^{1}$, Alexey S. Vlasov ${ }^{1}$ and Steven A. Blundell \\ ${ }^{1}$ Ioffe Institute, Saint Petersburg 194021, Russia; amintairov@gmail.com \\ 2 Electrical Engineering, University of Notre Dame, Notre Dame, Indiana 46556, USA \\ ${ }^{3}$ University Grenoble Alpes, CEA, CNRS, IRIG, SyMMES, F-38000 Grenoble \\ * Correspondence: amintairov@gmail.com
}

\begin{abstract}
In magneto-photoluminescence (magneto-PL) spectra of quasi-two dimensional islands (quantum dots) having seven electrons and Wigner-Seitz radius $r_{s} \sim 1.5$, we revealed a suppression of magnetic field $(B)$ dispersion, paramagnetic shifts and jumps of the energy of the emission components for filling factors $v>1(B<10 \mathrm{~T})$. Additionally, we observed $B$-hysteresis of the jumps and a dependence of all these anomalous features on $r_{s}$. Using a theoretical description of the magneto-PL spectra and an analysis of the electronic structure of these dots based on the single-particle FockDarwin spectrum and many-particle configuration-interaction calculations, we show that these observations can be described by the $r_{s}$-dependent formation of the anyon (magneto-electron) composites (ACs) involving single-particle states having non-zero angular momentum and that the anyon states observed involve Majorana modes (MMs), including zero- $B$ modes having equal number of vortexes and anti-vortexes, which can be considered as Majorana anyons. We show that the paramagnetic shift corresponds to a destruction of the equilibrium self-formed $v 5 / 2$ AC by the external magnetic field and that the jumps and their hysteresis can be described in terms of Majorana qubit states controlled by $B$ and $r_{s}$. Our results show a critical role of quantum confinement in the formation of magneto-electrons and implies the liquid-crystal nature of fractional quantum Hall effect states, Majorana anyon origin of the states having even $v$, i.e., composite fermions, which provide new opportunities for topological quantum computing.
\end{abstract}

Keywords: quantum dots; magneto-photoluminescence; fractional quantum Hall effect, anyons, Majorana modes, topological quantum computing.

\section{Introduction}

Superconducting (dissipationless) transport corresponding to zero electrical resistance of the materials, discovered in 1913 [1], gave a quantum mechanical illustration of Newton's first law of motion, which states that moving material objects will conserve a constant velocity if no external force acts on them, and which represents an obvious paradox for our imagination, since natural movements observed in everyday life are subject to a frictional force and are slowed down. Thus, in superconducting materials the persistent currents loops (PCLs) can be thought to be perpetual and laboratory measurements shows their life-time to be at least 100000 years [2]. In an external magnetic field $\left(B_{e}\right)$, the persistent vortex currents induce a perfect diamagnetism, i.e., the expulsion of $B_{e}$ from the superconductor (SC), known as the Meissner effect [3]. PCLs in SCs provide the quantization of the magnetic flux [4] in multiples of $\phi_{0}^{*}=h / 2 e_{0}$, where $e_{0}$ is the electron charge, which was documented in the measurements of the magnetization of micrometer size SC cylinders $[5,6]$. Single $\phi_{0}^{*}$ vortexes, also called Abrikosov vortexes or fluxons, arranged in regular lattices, are generated in major SCs above the critical field $B_{e 1}[7,8]$. The vortexes consist of tens of nanometers size PCLs, their density is proportional to $B_{e}$ and they merge at a critical field $B_{e 2}>B_{e 1}$, transferring the material into a resistive state. Thus, the existence of $\phi 0^{*}$ is directly related to the existence of systems having SC-type dissipationless transport. Such transport corresponds to a suppression of scattering ("friction") of charged carriers and exists in systems having a gap in the density of conducting states, 
which in conventional SCs arises from the formation of the bound electron pair state known as a Cooper-pair [9].

While it is commonly accepted to associate superconductivity with Cooper-type electron pairing, a zero electrical resistance, i.e., SC-type dissipationless transport, is observed in two-dimensional (2D) electron (e) gas semiconductor structures in the quantum Hall effect, i.e., at high perpendicular $B_{e}$, at integer (IQHE) [10] and fractional (FQHE) [11] Landau level (LL) filling factors $v=n \phi_{0} / B_{e}$, where $\phi_{0}=2 \phi_{0}^{*}$ and $n$ is the electron density. This transport is provided by a skipping-type edge current [12], which for the FQHE involves composite quasi-particles, called composite fermions (CFs) [13], consisting of $e$ with $1 / v$ magnetic-flux-quanta vortexes $(\mathrm{Vs})$ attached, resulting in a fractional charge equal to veo. The presence of $V \mathrm{~s}$ and SC-type gapped state, however, implies PCLs generated by CF $e$. While the necessity of such intrinsic sub-e PCLs was not considered in the commonly used description of the FQHE states, which is based on Laughlin's approach [14], their existence was revealed in our recent observation of FQHE-type states for single [15] and five [16] electrons confined in quasi-2D InP/GaInP2 islands-quantum dots (QDs) having dimensionless Wigner-Seitz radius $r_{\mathrm{s}} \sim 4$ and $\sim 2$, respectively, where $r_{\mathrm{s}}=1 /\left[a_{\mathrm{B}}{ }^{*}(\pi n)^{0.5}\right]$ and $a_{\mathrm{B}}{ }^{*}$ is a Bohr radius.

The FQHE-type states have been observed in these QDs at zero $B_{e}$ using high-spatialresolution magneto-photoluminescence (magneto-PL) spectroscopy in the measurements of the $B_{e}$-dispersion of PL spectral components and their spatial localization. The appearance of these states results in a built-in magnetic field $B \sim 6-15 \mathrm{~T}$ and a dependence of the ground-state, equilibrium fractional charge weo on the dot size $D$, for which 10 decreases with $D$. These observations imply a self-generation of $V \mathrm{~s}$ in a quantum state $|i\rangle$ occupied by a single $e$, and a fixed size of the $V$ (at zero external field $B_{e}$ ) equal to $2 a B^{*}$. The $e$, thus, can move without dissipation within a distance $r_{\mathrm{s}} a_{B}{ }^{*}$ and for $r_{\mathrm{s}}>1$ can be considered as nano-superconducting puddle, having charge-density distribution $\rho(\mathbf{r})=\left|\psi_{i}(\mathbf{r})\right|^{2}$, where $\psi_{i}$ is the wave-function of the state $|i\rangle$. Thus, $\psi_{i}$ can be formally considered to be a complex SC order parameter of the linearized Ginzburg-Landau equation [17] used for the description of mesoscopic SC structures $[18,19]$. This single-e-puddle can support $\sim r_{\mathrm{s}}$ sub-e PCLs generating $1 / 10 \mathrm{Vs}$. This reduces the Coulomb interaction energy of $e s$ and, thus, the state having $1 / 10$ sub-e PCLs and charge weo is self-formed [16]. The $w$ and corresponding $B_{v}=n 2 \phi_{0} w_{0}$ are directly determined by the dot size $(D)$ and the number of es $(N)$, i.e., $r_{s}$. We called such a state a magneto-electron $(\mathrm{em})$ and denoted it $e^{v}$, where the $\mathrm{em}$ composition $v=n / k$, with $n(k)$ the number of $e s(V s)$, is the quantity that substitutes the LL filling factor of the FQHE for quantum confined es. We observed ems having $v<1, n=1-4$, $k=1-9$ and molecular structures $6 e^{1 / 4}, 5 e^{1 / 4}, 3 e^{2 / 7}$ and $3 e^{1 / 3}+e^{4 / 15}$, having $e^{v}$ size $\sim 40 \mathrm{~nm}$ and bond length $\sim 60 \mathrm{~nm}$ for $N=5$ and 6 . The last two structures are transformed into one another by photoexcitation involving braiding and fusion of $e^{v} \mathrm{~s}$, which are the elementary topological quantum computing (TQC) operations [20].

We should point out that our observations of the fractional charge and the selfformation of $V \mathrm{~s}$ of a single $e$ at zero magnetic field sound like a paradox (similar to the perpetual persistent current in SC) or an artifact, not taking into account the quantum nature of single $e$ states, for which non-zero angular momentum states consist of "fractional charge" parts, and relying on the famous experiment of Millikan demonstrating a "fixed" $e_{0}$ value in charged oil droplets [21]. However, at the same time, in parallel experiments made by Ehrenhaft much smaller charges, i.e. sub-es, have been revealed in metal $(\mathrm{Au}, \mathrm{Pt}, \ldots)$ particles (see description of Millikan-Ehrenhaft dispute on sub-e in Ref. 22). Also, much later, a transfer of $1 / 3 e_{0}$ charge from tungsten to $\mathrm{Nb}$ balls has been detected in experiments on superconducting magnetic levitation [23]. We can suppose that these observations can be connected to our observation of $\mathrm{em}$.

Nevertheless, since we observed $e^{v}$ ems in QDs having $v<1$, one can expect their formation in QDs having $v>1$, as for FQHE states [24]. These states correspond to partial (total) filling of the highest (lower) LLs and involve CFs, which are anyons [16], in the highest LL and IQHE es in the lower one. 
A much investigated $v>1 \mathrm{FQHE}$ state is the $v=5 / 2$ state, which, according to the conventional description, corresponds to the half-filled second LL and fully filled lowest one. The CFs in the second LL show non-Abelian anyon properties [25], which implies that they have half-flux Vs with a zero-energy excited state, known as a Majorana zero mode or simply a Majorana mode (MM) [26-29], and, thus, are a perspective for the realization of TQC [30,31].

In $\mathrm{em}$ the $\mathrm{MM}$ is a particle-antiparticle pair, consisting of $V \mathrm{~s}$ having opposite, antiparallel direction/orientation. It can be naturally generated in a state having non-zero angular momentum $l_{z}$, for which the $e$-wave-function has "anti-phase spatial splitting". In such states, only half of the $\psi_{i}$ can generate $V$ along the $B_{e}$ direction, which thus is a half$V$ having a MM excited state. For the em the MM adds two more $V \mathrm{~s}$ resulting in a twotimes smaller charge, the signature of which was observed for the 5/2 state [32]. Moreover, since a MM adds zero magnetic flux, it can possibly be generated at zero $B$.

A realization of self-formed $v>1$ ems/anyons, in general, and $v=5 / 2$, in particular, requires a decrease of $r_{\mathrm{s}}$. In our earlier studies [33], we have measured a quantum-Halltype $\mathrm{InP} / \mathrm{GaInP} \mathrm{P}_{2}$ QD having $N \sim 9, r_{\mathrm{s}} \sim 1.5$ and $B \sim 2 \mathrm{~T}$, which approximately matches that expected for $\omega=5 / 2$.

Here we analyze a few such dots in detail using measurements of the $B_{e}$-dispersion of their PL spectra components. In the measurements, we have revealed several anomalies, which include suppression of the dispersion, paramagnetic shifts, jumps (up to $\sim 2$ $\mathrm{meV}$ ) and their hysteresis. We found that the appearance of these anomalies depends on the dot size and the direction of change of $B$. We analyzed the experimental data using a theoretical description of PL spectra and the electronic structure of such dots based on single-particle Fock-Darwin (FD) states and a many-particle configuration-interaction (CI) approach, which allows one to explain the observed anomalies by self-formation of $\mathrm{em}$ $\mathrm{MM}$-anyon composites corresponding to $w \sim 5 / 2$, their collapse and the emergence of the anti-em-MM composites induced by the magnetic field. Our analysis has shown that the formation of the ems corresponds to the generation of $V \mathrm{~s}$ by single and pair non-interacting es occupying quantum-confined states. This allows one to describe the FQHE in terms of liquid-crystal states involving Majorana anyons and opens new routes for the realization of topological quantum computing.

\section{Materials and Methods}

\subsection{Natural quantum Hall puddles}

The details of the growth procedure, structural and emission properties of the $\mathrm{InP} / \mathrm{GaInP}_{2}$ QDs were described previously [34]. The dots have a flat lens shape (aspect ratio 10) and a lateral size $D \sim 50-180 \mathrm{~nm}$. Their shape reveals a small elongation and asymmetry, which in most cases can be described as a combination of a $\sim 5 \%$ elliptical distortion $\left(D_{\|} / D_{\perp}=1.05\right)$ and a $10 \%$ change of $R_{\perp}$. The specific structural property of this QD system is an atomic ordering of the GaInP2 matrix material, which results in composite core-shell structure consisting of InP QD having zinc blende crystal structure surrounded by a few atomically ordered $\mathrm{GaInP}_{2}$ domains having rhombohedral crystal structure and size 10-50 $\mathrm{nm}$ [35]; in this composite the domains generate strong piezo-electric fields [36] resulting in $e$ doping (up to 20) and $B_{v}$ (up to $15 \mathrm{~T}$ ) forming natural quantum Hall puddles.

Here we studied three of such puddles denoted D01m, D07m and D09m. They have a number of electrons $N=6$ and 7 and $D \sim 75 \mathrm{~nm}$ resulted in the electron density $n \sim 3 * 10^{11}$ and $r_{\mathrm{s}} \sim 1.3$, which corresponds to a weak Wigner localization regime.

\subsection{Single dot magneto-photoluminescence spectroscopy measurements}

We have measured magneto-PL spectra of single puddles using a near-field scanning optical microscope (NSOM) having spatial resolution up to $25 \mathrm{~nm}$ operating at $10 \mathrm{~K}$ and magnetic fields of up to $10 \mathrm{~T}$ as described in detail in Refs. $16,33,34$. $\sigma^{+}$and $\sigma$-circular polarization were measured using $\lambda / 4$ plate and linear polarizer. In the spectra we measured the shifts of PL peaks related to occupied $e$-shells versus $B_{e}$, which were compared 
with the theoretical calculations and used to extract a charge of single particle states of the puddles.

\subsection{Analysis of the data}

The shape of the PL spectra, i.e., the number of spectral components, their position and full-width-at-half-maxima, were analyzed using a multi-peak fitting procedure from Origin 20.0 graphic software.

The values of effective (screened) quantum confinement $\hbar \omega_{0}{ }^{*}$ (see Appendix A.3) and $N$ were measured from the splitting and the number of anti-Stokes peaks in the PL spectra, respectively. For $N$ the measurements the shifts of anti-Stokes components in magnetic field were used as well. The $D$ values were estimated from the charge density distributions (CDD) calculated from the unscreened quantum confinement value $\hbar \omega_{0}=\hbar \omega_{0}^{*} / 0.7$ and $N$ values using CI approach $[16,34]$ and from NSOM scanning experiments. Using $N$ and $D$ values we calculate $n$ and $r$ s. Note, that these values are related to the photo-excited state having $N^{*}=N+1$ electrons.

\subsection{Theory and calculations}

The theoretical description of the PL spectra of quantum Hall puddles in a magnetic field based on the Fock-Darwin (FD) spectrum is presented in Appendix A. The description involves a general model (Appendix A.1), an analysis of the single-particle states of $\mathrm{InP} / \mathrm{GaInP}_{2} \mathrm{QDs}$ involved in PL transitions using the 8 band $\mathbf{k} \cdot \mathbf{p}$ method, i.e., non-interacting electrons and holes (Appendix A.2), and the analysis of the effect of the Coulomb interaction on single-particle states using the Hartree-Fock (HF) method (Appendix A.3). The resulting formulas of the model, i.e., $B_{e}-$ dispersion of PL peaks, are Equations A4c and $\mathrm{A} 5$ in Appendix A.4.

The ground states (GSs) of the puddles in a magnetic field were analyzed in Appendix $B$. Total energy and total angular momentum values were calculated using the multiparticle CI method (Appendix B.1) and the single-particle FD spectrum including the vortex contribution (Appendix B.2). Both methods revealed GSs having fractional v, suggesting em-composite (em-C) formation.

The parameters of $e m-C s$, which are composition $v_{n}{ }^{+}\left(v_{n}\right) s$, where $n$ is number and $S$ is a total spin, and charge $e_{N}{ }^{*}$ are specified in Appendix C.1 (see Equations C1-C3). There a mismatch of the emission energy of em-Cs is analyzed and corresponding quantities, which are $\Delta_{v}$ jumps and related PL shift $\left(E_{s}{ }^{m-C}\right)$, are described (Equations C4 and C5).

The fitting of Fock-Darwin spectrum for the measurements of $v$ and details of construction of $V$ structure of em-Cs are described in Appendix C.2 and C.3, respectively.

\subsection{Summary of measured parameters}

In Table 1, we summarize the initial zero-field parameters of the puddles, which are the number of the electrons in photo-excited state $N^{*}=N+1, D, r_{s}, \hbar \omega_{0}^{*} / \hbar \omega_{0}$, and the final parameters of $\mathrm{em}$-Cs formed in these puddles obtained from the measurements and the analysis of the data, which are two intrinsic magnetic fields $B_{\mathrm{ec}}$ and $B_{v}$ (see below), and $\nu_{0}\left(\nu^{+}\right) s$ and $v_{n}\left(v_{n}+\right) s$ values of the equilibrium and $B_{e}$-induced em-Cs, respectively. In the notation of the compositions in Table 1, we include reduced charge value $e_{N}{ }^{*}$ as a superscript. 
Table 1. Properties of anyon(magneto-electron) composites of $\mathrm{InP} / \mathrm{GaInP}_{2}$ quantum Hall puddles.

\begin{tabular}{|c|c|c|c|c|c|c|c|c|c|}
\hline QD & $N^{*}$ & $\begin{array}{c}\hbar \boldsymbol{\omega}_{0}^{*} / \hbar \boldsymbol{\omega}_{0} \\
(\mathrm{meV})\end{array}$ & $\begin{array}{c}D \\
(\mathrm{~nm})\end{array}$ & $r_{\mathrm{s}}$ & $\begin{array}{c}\boldsymbol{B}_{\mathrm{ec}} / \boldsymbol{B}_{\boldsymbol{v}} \\
(\mathrm{T})\end{array}$ & $\left.\mathrm{w}^{+}(\mathrm{w})\right)^{e N^{*}}$ & & $\boldsymbol{V}_{n}+\left(\boldsymbol{v}_{n}\right)_{S^{e N^{*}}}$ & \\
\hline D01m & 7 & $5.5 / 9.2$ & 65 & 1.2 & $3 / 3.6$ & $-5 / 2(-21 / 8) 3 / 2^{3.75}$ & $23 / 9(\infty) 1 / 2^{4.91}$ & $27 / 11(5 / 2) 3 / 2^{3.91}$ & - \\
\hline D07m & 8 & $4.5 / 7.5$ & 75 & 1.3 & $2.5 / 3.2$ & $-21 / 8(-8 / 3) 0^{3.05}$ & $8 / 3(\infty)_{1}^{5.66}$ & $5 / 2(5 / 2) 1^{5.41}$ & $10 / 7(7 / 4) 2^{4.25}$ \\
\hline D09m & 8 & $3.5 / 5.8$ & 85 & 1.5 & $2 / 3$ & $-9 / 4(-5 / 2) 0^{2.08}$ & $32 / 13(\infty) 1^{4.91}$ & $23 / 10(3) 2^{3.88}$ & $9 / 7(4 / 3) 2^{3.71}$ \\
\hline
\end{tabular}

\section{Experimental results}

\subsection{Shell structure and built-in magnetic field of quantum Hall puddles}

Figure 1a compares the PL spectra of the dots/puddles studied at zero internal field $B$ (see below), plotted in Stokes energy units. The inserts show CI CDDs of the dots and a contour plot of spatially resolved PL spectra near the center of D07m dot. Figure $1 \mathrm{~b}$ shows circularly polarized spectra of the D07m dot measured at $B_{e}=0,1,2, \ldots, 10 \mathrm{~T}$.

CDDs show a decrease of the size related to a decrease/increase of $D / \hbar \omega_{0}$ (see Table 1). The CDDs are elongated along $x$ and their landscape has the same topology consisting from two minima of $\sim D / 6$ size separated along $x$ by $\sim D / 3$, which indicates molecular structure (see Appendix B.1). The left insert shows the measured size of the emission area of the D07m dot of $\sim 90 \times 60 \mathrm{~nm}$ (average size of $75 \mathrm{~nm}$ ), in agreement with the corresponding CDD size.

In Figure 1a it is seen that the spectra of the dots studied consist of the main peak denoted by $s$, and two weaker anti-Stokes ones denoted by $p$ and $d$. These three peaks are related to three occupied e-shells of the QD in the photo-excited state, as described in Appendix A.1. The peaks have a full-width-at-half-maximum $\sim 3 \mathrm{meV}$ and varying splitting corresponding to $\hbar \omega_{0}^{*}$ (see Table 1), related to the size variation. The D09m dot also has a few times larger relative intensity of $p$ - and $d$-components. There is an increase of the ratio of the $s-p$ to $p-d$ splitting related to the size increase.
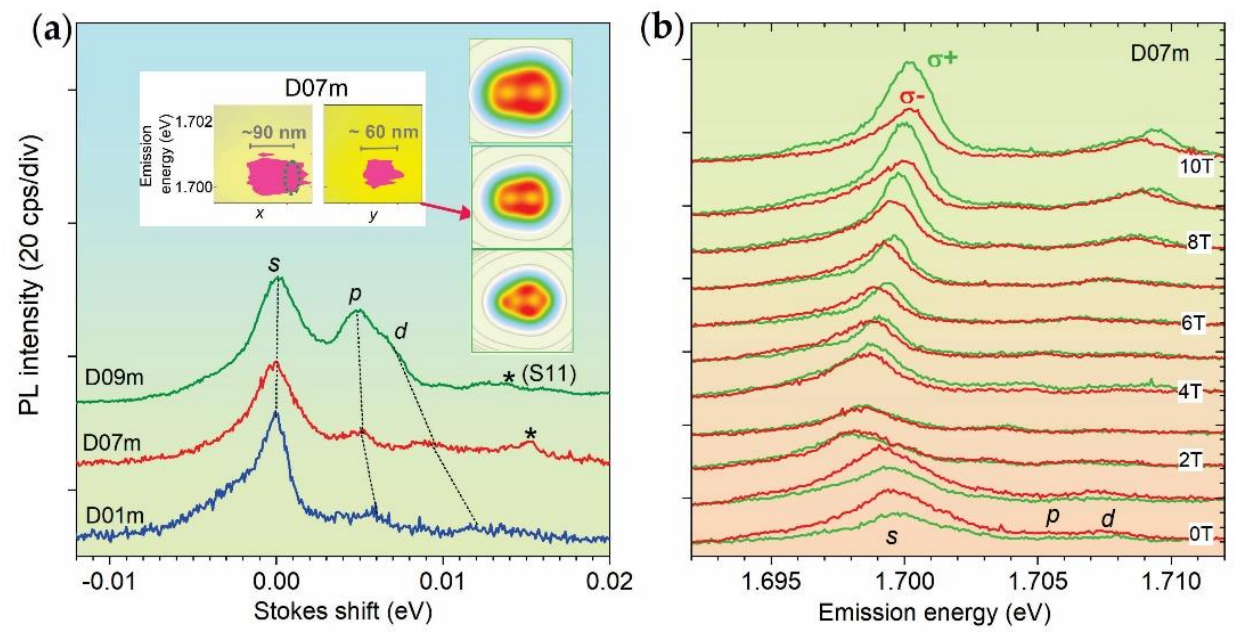

Figure. 1 (a) Comparison of low-temperature (10K) PL spectra of D01m, D07m and D09m $\mathrm{InP} / \mathrm{GaInP}_{2}$ QDs. Lines connect the peak maxima and stars denote neighboring dots. Center inserts show portions of contour plots of a set of spatially resolved near-field PL spectra of D07m measured along two perpendicular directions, in which regions corresponding to the size of emission area are outlined by the magenta color and the horizontal bars. The dashed ellipse in $x$-scan outlines contribution of neighboring dot. Right inserts show charge density distributions (size 100x100 nm) of the dots calculated using the CI method. (b) Circular polarized spectra of D07m measured in the range $B_{e}=0-10 \mathrm{~T}$. 
In Figure $1 b$ the PL spectra of the D07m dot at zero $B_{e}$ reveals nearly a two-times stronger intensity of the $\sigma^{-}$-component, i.e., they are $\sigma^{-}$-polarized, which indicates $B v$. At $B_{e c}=3 \mathrm{~T}$, the $\sigma^{-}$-polarization disappears and the spectra acquire a $\sigma^{+}$-polarization at larger $B_{e}$. This indicates $B_{v}-B_{\text {ec }}$ (see Table 1$)$, where $B_{\text {ec }}$ is a compensating field and $B_{v}$ has a direction opposite to $B_{e}$. Thus, in the range from $0 \mathrm{~T}$ to $B_{\mathrm{ec}}$ the internal field $B=B_{e}+B_{v}$ is negative and decreases from $B_{v}$ to zero. In this range, which we denote $B^{-} \downarrow$ or $B_{\mathrm{e}}{ }^{\mathrm{a}}, B_{e}<\left|B_{v}\right|$ and the PL spectra have an anomalous $B$ e-shift (see below). Such an anomalous shift is a direct signature of $B$, which allows one to detect it independently from polarization measurements. For larger fields, i.e., $B_{e}>\left|B_{v}\right|, B$ becomes positive and increases versus $B_{e}$. In this range, which we denote by $B^{+} \uparrow$ or $B_{\mathrm{e}^{\mathrm{n}}}$, normal $B_{\mathrm{e}}$-shifts are observed, but $B$ is lower $B_{e}$ on $B$ ec.

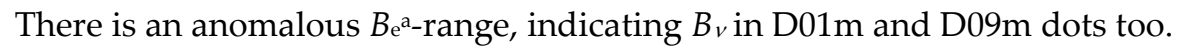

\subsection{Shell structure in magnetic field}

In Figures 2a-2c, we present unpolarized spectra of the dots, measured at $B_{e}=0,1,2$, $\ldots 10 \mathrm{~T}$. The spectra were measured under an increase of the field from 0 to $10 \mathrm{~T}$, denoted as $B_{e} \uparrow$ and shown in the lower part of the graphs, and a decrease from 10 to $0 \mathrm{~T}$, denoted as $B_{e} \downarrow$ and shown in the upper parts of the graphs. For this range, the $B \mathrm{e}^{\mathrm{n}}$ and $B \mathrm{e}^{\mathrm{a}}$ ranges are the $B^{+} \downarrow$ and $B^{-\uparrow}$-ranges, respectively.

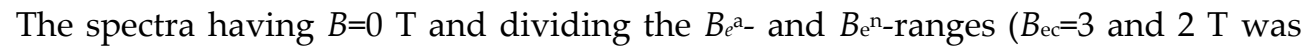
found in D01m and D09m dots, respectively) are shown by thick solid lines. In Figure 2a2c the peak maxima are connected by straight lines, which allows one to qualittatively trace their shift.

At all $B_{e}$ values the s-peak is dominant and changes of the spectral shape are caused by the changes of the intensity and the position of the $p$ - and $d$-peaks at $B_{e}>4$ T. These are: the peak merging at $B_{e}=7 \mathrm{~T}$ in the D01m dot; the few-times intensity increase of the $d$-peak at $B_{e}=9 \mathrm{~T}$ and the appearance of the additional $x$-peak between the $s$ - and $p$-peaks at $B_{e}=7$ $\mathrm{T}$ in D07m dot; a few-times intensity decrease of the $p$ - and $d$-peak at $B_{e}=8 \mathrm{~T}$ in the D09m dot.
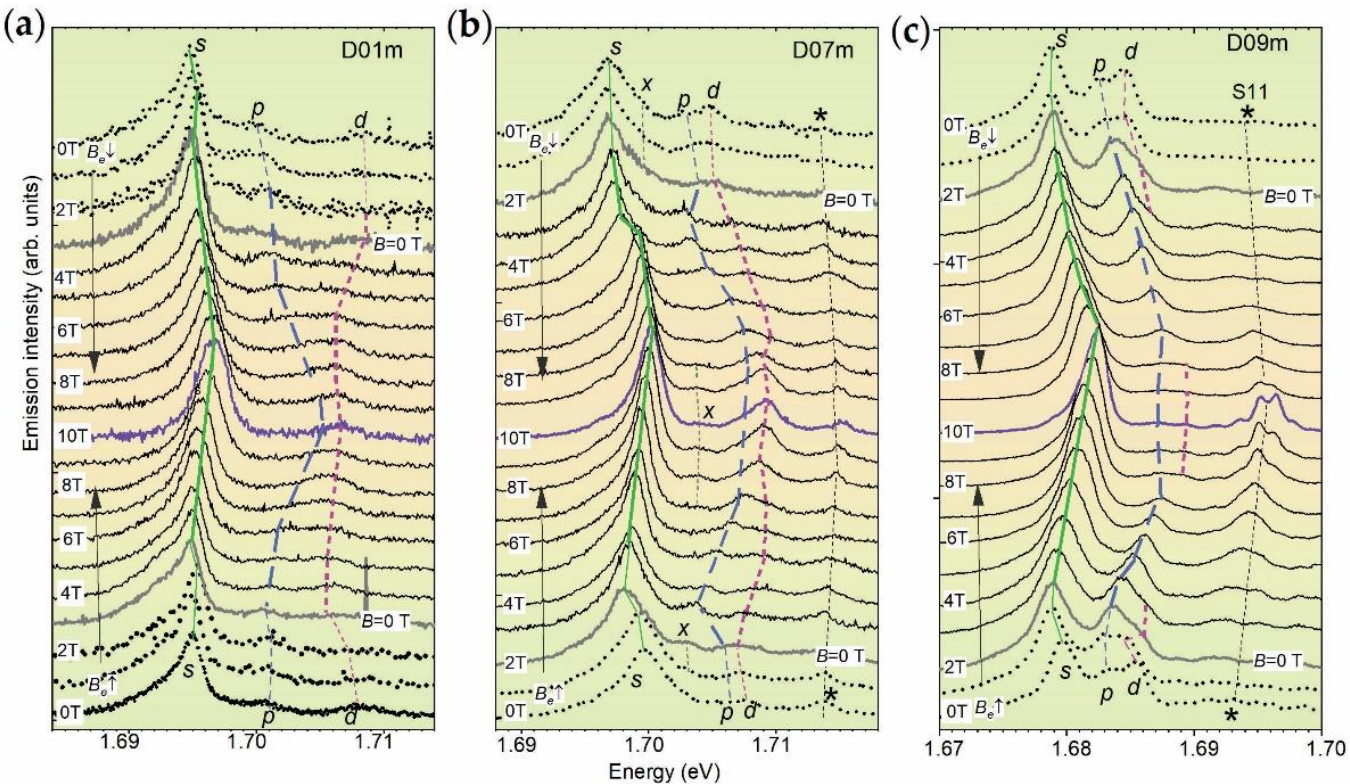

Figure. 2 Low-temperature (10K) magneto-PL spectra (thin solid curves) of D01m (a), D07m (b) and D09m (c) QDs measured in the field range $B_{e}=0-10 \mathrm{~T}$ (thick solid curves are for $B=0 \mathrm{~T}$, shortdot curves are for $\left|B_{e}\right|<\left|B_{\mathrm{ec}}\right|$ ). Solid, dashed and short-dashed lines (thinner for $\left|B_{e}\right|<\left|B_{\mathrm{ec}}\right|$ ) connecting the maxima of $s-, p$ - and $d$-peaks, respectively, are drawn to outline their shift and bends; a star denotes neighboring dot, which in (c) is the single electron dot S11 studied in Ref. 15. Vertical arrows at the left show direction of field increase. 
The peak shift, further denoted as $s, p$ - and $d$-shifts, in the $B e^{\mathrm{n}}$-range is mostly positive, i.e., increases with field increase, and at $B_{e}=10 \mathrm{~T}$ reaches average values of $\sim 2, \sim 5$ and $\sim 3$ $\mathrm{meV}$, respectively. In the $B_{\mathrm{e}} \mathrm{a}$-range the shifts are negative (paramagnetic), i.e., "anomalous", and have values from 0 to $2 \mathrm{meV}$. In both ranges the shifts reveal a few bends (see spectra near $B_{e} \sim 3 \mathrm{~T}$ and $6 \mathrm{~T}$ ).

\subsection{Anomalous shifts and jumps induced by magneto-electrons}

In Figures 3a-3c, we show the measured data points of the peak positions versus $B$ and calculated $B$-dispersion of the peaks overlaid on the contour plots of the spectra. Calculated shifts are presented in the $B \mathrm{e}^{\mathrm{n}}$-range, i.e., for $\mathrm{B} \geq 0$ T. The peaks are denoted by the quantum numbers of the Fock-Darwin (FD) spectrum $k l$ (see Appendix A.2).

The $s$-shift data show that the bends seen in the spectra in Figures 2a-2c are jumps having amplitude 1-2 meV over $\Delta B_{e} \sim 1 \mathrm{~T}$ (see dashed ovals in Figures 2a-2c). Their appearance is different in different dots and $B / B_{e}$ ranges, revealing size dependence and $B_{e} \uparrow-B_{e} \downarrow$ asymmetry (hysteresis). In the D01m dot, one step is observed in both $B-\downarrow-$ and $B^{-\uparrow}$-ranges at $B=-1 \mathrm{~T}$, which is thus symmetric. In the D07m dot, two asymmetric steps are observed, one in the $B^{-} \downarrow$ - and the other in the $B^{+} \downarrow$-ranges at $B=-2$ and $5 \mathrm{~T}$, respectively, and no mirror steps appear in the ranges of reversed direction of field change. In the D09m dot, four asymmetric steps are observed in the $B^{-} \downarrow$ - and $B^{+} \uparrow$-ranges at $B=-2,3,5$ and 7 .

The calculated s-shift shows a weak nearly linear dispersion with slope $\sim 0.25 \mathrm{meV} / \mathrm{T}$ and in the regions where the jumps are absent the experimental slope is the same as the calculated one.

The $p$-shift follows a dispersion of 10 -state up to $B_{c} \sim 5 \mathrm{~T}$ and at larger field the shift is saturated (for D01m dot) or becomes negative (for D07m and D09m) approximately reaching the 02-04 states, i.e. shows bending related to level crossing (see Figure B1c). The $p$-shift has a $\sim 30 \%$ smaller slope than the 01 -state $(\sim 1 \mathrm{meV} / \mathrm{T})$.

The $d$-shift follows the 11-state for $B_{e}<B_{c}$ for all dots and the 03-, 04- and 05-states for $B>B_{c}$ and the D01m, D07m and D09m dots, respectively, revealing a level crossing similar to the $p$-shift. For the D01m dot, the $d$-shift is strongly suppressed compared to the 11state.
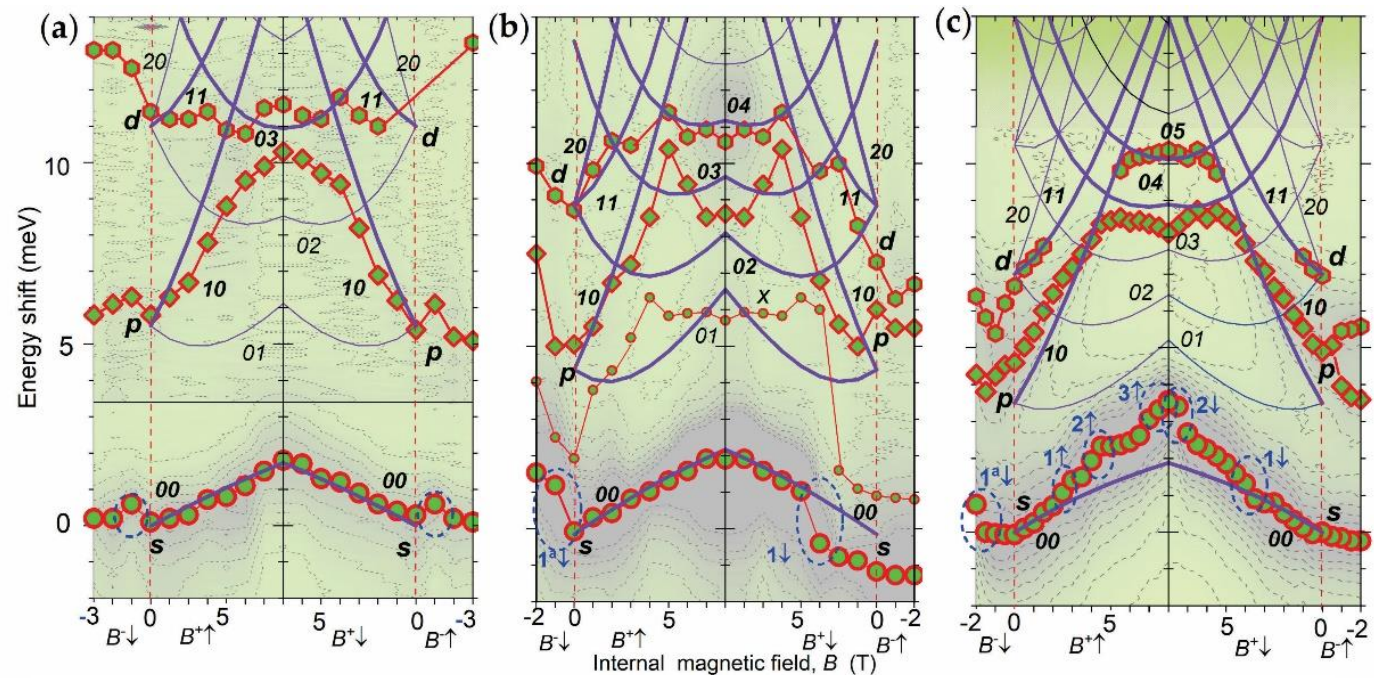

Figure 3. Peak shifts (circles $-s$, diamonds $-p$, hexagons $-d$, small circle $-x$ ) versus internal magnetic field Band calculated shifts (solid curves) overlaid on the PL spectra contour plot of D01m (a), D07m (b) and D09m (c) InP/GaInP2 QDs. Changes of $B$-field has four ranges: decrease from $B_{v}$ to $0 \mathrm{~T}\left(B^{-} \downarrow\right)$, increase from 0 to $10 \mathrm{~T}-\left|B_{\downarrow}\right|\left(B^{+} \uparrow\right)$, decrease from 10T$\left|B_{v}\right|$ to $0 \mathrm{~T}\left(B^{+} \downarrow\right)$ and increase from $0 \mathrm{~T}$ to $B_{v}\left(B^{-} \uparrow\right)$. Dashed ovals mark $s$-shift jumps.

In the $B_{\mathrm{e}}^{\mathrm{a}}$ range the experimental data points do not coincide with mirror counterparts in the $B \mathrm{e}^{\mathrm{n}}$-range, i.e., the shifts have a $B \mathrm{e}^{\mathrm{a}} / B_{\mathrm{e}^{\mathrm{n}}}$ asymmetry. This is most clearly seen for the $d$-peak in the D01m dot. 
The appearance of the $B e^{a}$-range is direct evidence for the existence of the ems in this range. For the $B e^{n}$-range, the existence of the ems follows from the observation of the jumps of the s-peak and the suppression of the $B_{e}$-dispersion of the $p$ - and $d$-peaks for $B_{e}<B_{c}$. The former are $\Delta_{v}$ steps and the latter is direct evidence for charge reduction of the $e$ in the single-particle state (see Appendix C.2).

Since the $B$ has opposite directions in the $B \mathrm{e}^{\mathrm{a}}$ and $B e^{\mathrm{n}}$ ranges, the $V \mathrm{~s}$ and $e m \mathrm{~s}$ of the former are anti-Vs (aVs) and anti-ems (aems) in the latter (see Appendix C.1). The ems at zero $B_{e}$ are self-organized and can be considered to be equilibrium ems, which we will denote as $S$-ems. Thus, the shifts of the peaks in the $B^{a}{ }^{a}$-range are related to the destruction of $S$-ems by the external field, which implies that the internal field generated by $S$-ems can be $B_{v} \neq B_{\text {ec }}$ and the $B$ decrease versus $B_{\mathrm{e}}$ increase in this case is non-linear in the $B_{\mathrm{e}} \mathrm{a}^{-}$range, as observed. The $e m s$ in the $B e^{\mathrm{n}}$ range are induced by the $B_{e}$ and can be considered as stimulated, further denoted as B-ems.

\subsection{Majorana anyon composites in Fock-Darwin spectrum}

In Figures $4 \mathrm{a}-4 \mathrm{c}$, we present the results of the e-em-FD spectrum fit to the experimental data. The data and fit are presented in the reduced field $\mathrm{VB}^{-1}$ and energy $\omega / \omega_{0}$ units (see Figure B1c) and the $e$-em-FD-states (e-em-FDSs) are labeled as $\mathrm{e}^{v_{l}}$, where $l$ is angular momentum of the state (see Appendix C.1). In the figures we also show total charge values $e_{N}^{*}$ (upper plots) and constructed $V$-structures (inserts). Total charge values are shown for photo-exited and initial states and were used to calculate the $\Delta_{v}$ and $E_{S}{ }^{e m-C}$. The experimental data for $B_{e} \downarrow$ and $B_{e} \uparrow$ measurements are plotted in the same graph and they reveal a $B_{e} \uparrow-B_{e} \downarrow$ asymmetry of the s-peak jumps as discussed above, and the same for $p$-shift in D07m and D09m for $v^{-1} \sim 0$ and 0.4-0.9, respectively.

A comparison of the plots in Figures $4 \mathrm{a}-4 \mathrm{c}$ shows different $v^{-1}$ ranges, acquired using the same $B_{e}$ range, in the dots studied, which is due to the different dot size giving an increase of the $v=1$ field $B_{0}$ as $D$ decreases (see Appendix B.1). The final $v^{-1}$ value is 0.6 for the smallest D01m dot and 1.1 for the largest D09m dot. At the same time, the $v_{B}{ }^{-1}$ starting value is the same $\sim-0.29$, i.e., $v^{\sim-7 / 2}$.

Also, a comparison of the plots shows that the $\mathrm{vB}^{-1}$-dispersion of the FDSs is smoothed out as $D$ increases (see the shallowing of $2 e_{1}$ level "depth" at $v^{-1} \sim 0.5$ ), which shows a suppression of the quantum confinement effect.

The data in Figures $4 \mathrm{a}-4 \mathrm{c}$ show that the ems are observed in the $p$ - and $d$-single particle states forming $B$-em-Cs over the entire $B e^{n}$-range measured. The composites correspond to a set of $n$ GSs $\mid v_{n s}{ }^{+}>N_{1 / v B}$ given in Table 1. In all B-em-Cs, only the $2 e$ s-state is $e m$-free and has $v=2$ ( $e^{2} 0$-state) for $n=1$ and 2 and $v=1\left(2 e^{1} 0\right.$-state) for the $n=3$ composites. The rest are $B$-em states, which have $v=1$ and $2 / 3$ for $2 e p_{y} / p_{x}$-states and $v=1 / 2,1 / 3,14$ and $1 / 8$ for $1 e 10\left(p_{x}\right)-, 02\left(d_{y}\right)-, 20\left(d_{x}\right)$ - and $03\left(f_{y}\right)$-states, depending on $B, N^{*}$ and $D$.

The $n=1$ structures in the inserts in Figures $4 \mathrm{a}-4 \mathrm{c}$ show that in the D01m dot B-em $\mathrm{M}_{-} \mathrm{C}$ has two single-state Majorana ems $\left(e m^{\mathrm{M}}\right)$ (see Appendix C.1) $e^{1}-1(0)$ and $e^{1 / 4}-2(0)$ and one two-state $e m^{\mathrm{M}}$, formed by $s-e$ and $e^{2 / 3_{1}}$ states, in which the $V$ of the former is compensated by the $a V$ of the latter. Note, however, that this compensation is not complete since two $s$ es in the $v=2$ state generate two-times smaller flux. In the D07m dot, the $n=1 \mathrm{em}^{\mathrm{M}} \mathrm{C}$ has the same number of $V_{\mathrm{s}} N_{V}$ since the extra $e$ generates $e^{1 / 2} 2_{2}(0) e m^{\mathrm{M}}$ in a $d_{\mathrm{x}}$-state taking two $V \mathrm{~s}$ from the $d_{\mathrm{y}}$-state. In the D09m dot, MMs $e^{1 / 2}-2(2)$ and $\left.e^{1 / 2} 2_{2}\right)$ are generated in $d_{\mathrm{x}}$ - and $d_{\mathrm{y}}-$ states increasing the $N_{V}$ on four $V \mathrm{~s} / a V \mathrm{~s}$.

For $n=2$ and $n=3 \mathrm{em}^{\mathrm{M}}-\mathrm{C}$ are formed by addition of $V \mathrm{~s}$ and elimination of $a V \mathrm{~s}$ resulting in an increase/decrease of $N_{V} / v_{n} S^{+}$. Thus, we observed the expected $N_{V}$ increase, with an 


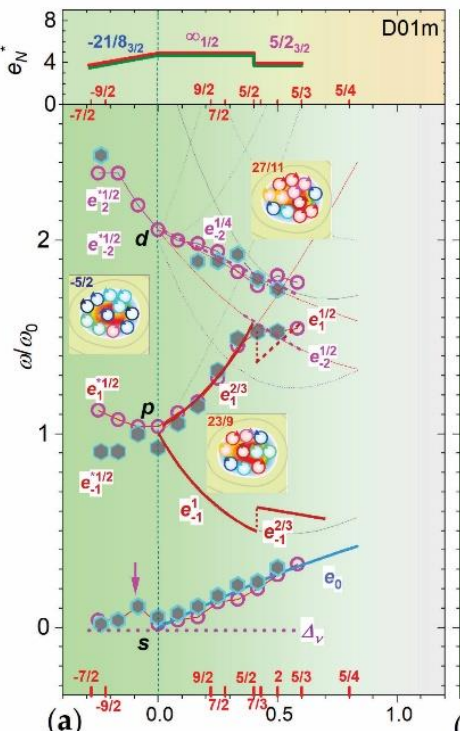

(a)
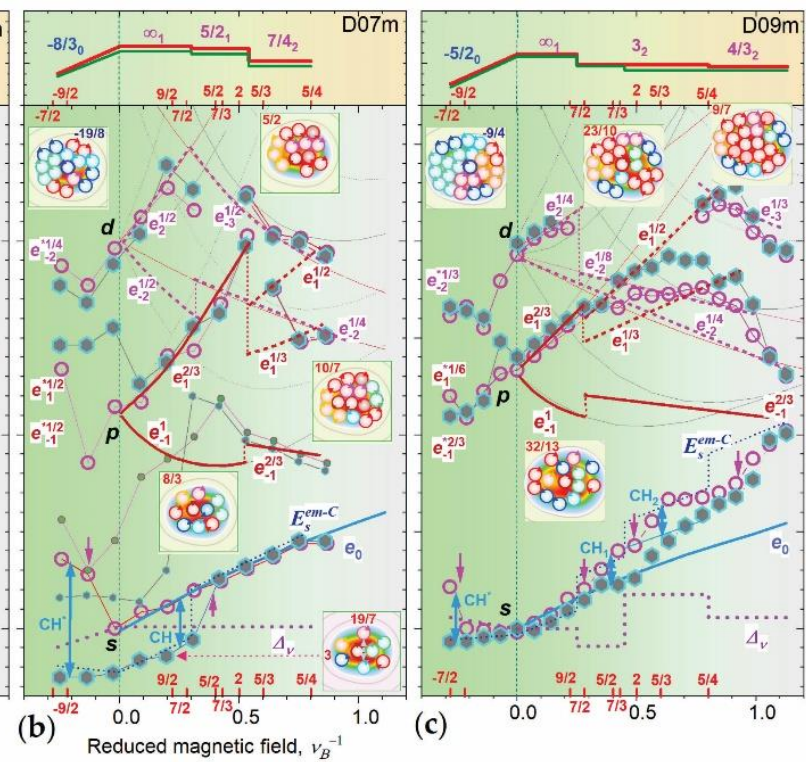

Figure 4.. Experimental (empty circles/solid hexagons are for $B_{e} \uparrow / B_{e} \downarrow$ measurements) and

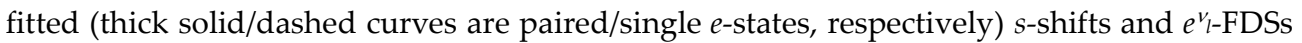
(lower plots) and $e_{N^{*}}^{*}$ (upper plots) versus $\mathcal{V}^{-1}$ for D01m (a), D07m (b) and D09m (c) $\mathrm{InP} / \mathrm{GaInP}_{2}$ QDs. Insets are $V$-structures of the $\mathrm{em}$-Cs represented by $N_{V} V$ s arrangement (arrowed circles) overlaid on the dot CDD. Numbers on $v^{-1}$ axis, total charge curves and inside insert's frames are $v, v_{n} s^{+}$and $v_{n}$ values, respectively. Arrows (one/double sided), thick and thin dotted curves on $s$-shifts are psotion/hysteresis of the jumps, $\Delta_{v}$ and $E_{s}{ }^{e m-C}$ functions, respectively (see text). Vs circles color code is for B-ems - dark red, yellow/violet and red are $V s$ in $s^{-}, p$ - and $d$ states and light blue/green and blue are $a V \mathrm{~s}$ in $p$ - and $d$-states; for $S$-ems the colors for $V \mathrm{~s}$ and $a V s$ are interchanged. The insert at the lower right corner of $(\mathbf{b})$ is the $V$-structure of $n=1$ in initial state in $B_{e} \downarrow$-range in which dashed circles, marked by arrows, correspond 19/7 state (see

increase of $D$ and $B$, and the minimum $N_{V}$ value of nine has $n=1 \mathrm{em}^{\mathrm{M}}-\mathrm{C}$ in $D 01 \mathrm{~m}$, while the maximum of twenty one has $n=3 \mathrm{em}-\mathrm{C}$ in D $09 \mathrm{~m}$.

The charge values obtained are in the range $e_{N}=(3-6) e_{0}$, which is thus up to two times smaller than that without ems, i.e., (7-8)eo. The reduced charge for $n=1$ corresponds to a zero field $B_{v}$ generated by the composite, i.e. $n s^{+}=\infty$, and thus can be considered as Majorana anyon/em-C $\left(\mathrm{em}^{\mathrm{M}}-\mathrm{C}\right)$. This $\mathrm{em}^{\mathrm{M}}-\mathrm{C}$ has $\mathrm{es}^{*}=4.91,5.66$ and 4.91 in the dots D01m, D07m and D09m, respectively, which shows that the increase of $e_{N}$ of D07m relative to D01m due to an extra $e$ is compensated in D09m by its decrease due to the increase of the $N v$.

\subsection{Charge hysteresis and Majorana modes}

The $\Delta_{V}$ values calculated show an absence of the steps in the D01m and D07m dots and their presence in the D09m dot (see the corresponding curves in Figures 4a-4c). Zero $\Delta_{V}$ (no steps) occurs when the photo-excited (topmost) em states and the initial states of the neighboring em-Cs have the same $v$ and $N v$, respectively (see Equation C4). This is the case for the smallest D01m dot for both $B_{e} \uparrow$ and $B_{e} \downarrow$ measurements.

For the intermediate size D07m dot, this is took place only in the $B_{e} \uparrow$ data, and in the

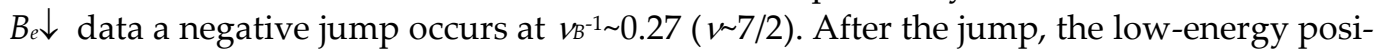
tion of the s-peak is maintained under a further field decrease down to zero. This shows that two $\mathrm{em}^{\mathrm{M}}$-Cs exists in the initial state in the D07m dot, which are $3(\infty)$ and $19 / 7(\infty) \mathrm{em}^{\mathrm{M}}$ Cs (see $V$-structure in the lower right insert in Figure $4 \mathrm{~b}$ ), among which the latter is a MM of the former appearing because of the generation of an $e^{1}-1(0) e \mathrm{~m}^{\mathrm{M}}$. The $B_{e} \downarrow$-jump is thus related to a charge increase in the initial state due to $N_{V}$ reduction in the $3(\infty)-\mathrm{em}^{\mathrm{M}}-\mathrm{C}$ and is reproduced well by $E^{e m-C_{\mathrm{s}}}(B)$. Thus, $B_{e} \uparrow-B_{e} \downarrow$ measurements reveal charge hysteresis

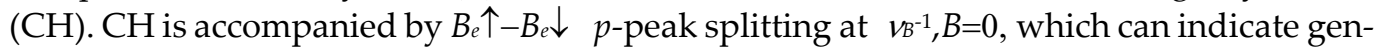
eration of additional $V \mathrm{~s}$ in the $p$-state.

In the largest dot D09m the $u$ values of the four topmost em states are $1 / 4_{2}, 1 / 8-2,1 / 2_{1}$ and 1/3-3, which are different and thus give three $\Delta_{\nu}$ steps at $v=7 / 2,2$ and $5 / 4$, the first and 
the third of which are negative, as is seen in Figure 4c. The number and position of these steps match well to that of the $B_{e} \uparrow$ experiment, but the sign of the first and third jumps is positive instead. This implies a MM in the corresponding initial state, substituting Vs of the photo-excited em. Analysis has shown that this is the $\mathrm{e}^{1}-1(0) \mathrm{em}^{\mathrm{M}}$ (the same as in the D07m dot) forming the $e^{2 / 5_{-}}(1) e m$ from the $\mathrm{e}^{2 / 3} \mathrm{~s}_{-1}(1)$ one. The calculated $E_{\mathrm{s}}{ }^{\mathrm{em}-\mathrm{C}}$ accounting for this MM shows good agreement. The $B e \downarrow$ data shows two $\mathrm{CH}$ starting at $v \sim 5 / 4\left(\mathrm{CH}_{1}\right)$ and $2\left(\mathrm{CH}_{2}\right)$, respectively. In the $\mathrm{CH}_{2} \mathrm{em}$ - $\mathrm{C}$ there is an increase of $p$-peak energy indicating generation of additional $V \mathrm{~s}$.

\subsection{Collapse of S-magneto-electron-composites}

The structure of $S$-em-Cs and the $v^{+}\left(B_{v}\right)$ values can be derived from the crossing of the positions of the $p$ - and $d$-peaks at $B_{e}=0$ and $e m$-FDSs of the $B_{n}$-range. Thus, a position of the topmost $d$-peak corresponds to a $d_{x}$-state at $v \sim 5 / 2$ and $S=3 / 2$ in the D01m dot and to $d_{y}$-state at $v \sim 5 / 2$ and $S=0$ in the D07m and D09m dots. Note, that, while for the D01m dot the total spin of the mirror aem $S$ - and B-composites is the same (3/2), for the D07m and D09m dots it is different ( 0 versus 1 and 2). Note, also that the $w^{+}$value of all dots (see Table 1) are nearly the same $\sim 5 / 2$ (for dots D $01 \mathrm{~m}$ and D07m $\nu^{+}=5 / 2+1 / 8$ and $5 / 2+1 / 6$, respectively) and the corresponding $B_{v}$ values are larger than $B_{e c}$ on $\sim 1 \mathrm{~T}$.

The preference of the self-formation of the $S$-em-Cs follows from their smaller charge compared to the B-em-Cs; it is $3.75 e_{0}, 3.05 e_{0}$ and $2.08 e_{0}$ for the $S$-em-Cs versus 3.91e0, 5.41e and $3.85 e$ for the $B-e m-C s$ for the D01m, D07m and D09m dots, respectively. Charge reduction is provided by the extra $V \mathrm{~s}$ in the $p$-states (see the corresponding $V$-structures in the left inserts in Figures 4a-4c) and thus there can be some mechanism for selecting the $V$ direction in these states. These preferential $V \mathrm{~s}$ create $e^{*_{1} / 2_{-1}(2)}$ and $e^{* 1 / 6}(4) S$-ems (we add a superscript * to distinguish $S$-ems from B-ems).

In the D01m dot a symmetric positive $s$-jump at $v^{-1} \sim 0.1$ related to $S$-em-C destruction indicates the generation of $a V \mathrm{~s} / V \mathrm{~s}$ decreasing/increasing $B_{v}$ needed for formation of $n=1$ $B-e m-C / S-e m-C$ in $B_{e} \uparrow / B_{e} \downarrow$ measurements. A transition to S-em-C for the $B_{e} \downarrow$ data is accompanied by a red shift of the $p$-peak indicating emission of the $p_{y}$-component, which is not seen for $B-e m-C s$. Its appearance implies a redistribution of the hole density (see section A.2) under $V$ formation and the instability of $B-\mathrm{em}-\mathrm{C}$.

In the D07m and D09m dots negative $B_{e} \uparrow s$-jumps correspond to annihilation of MMs under a decrease of $B$ and their absence in the $B \downarrow \downarrow$ data indicate a suppression of the MM under the collapse of $n=1 B$-em-Cs. The signatures of S-em-C instability in these dots is a $B e \uparrow-B e \downarrow$ splitting of the $p$-peak, which is stronger in D07m dot.

\section{Discussion}

\subsection{Majorana anyons}

A subset of the GSs measured is different from that obtained in the exact quantum mechanical CI calculations in Appendix B.1. For D09m dot they are $\mid 8_{0}, 7 / 2_{1}, 7 / 3_{0}, 21>8_{0.6}$ (see Table 1) and $\left.\right|_{\infty}, 3_{2}, 4 / 3_{2}>8_{1.1}$ (see Figures B1a and B1b), respectively, showing a reduction of the number of $B$-em-C GSs compared to $e$-GSs due to a suppression of the level crossing (see Appendix C.2). The CI set includes fractional $v$ GSs, which, however, do not imply a generation of $V$ s by single electrons, i.e., fractional charge, and reflect only a matching of the number of $V s$ to the total angular momentum value [37, 38]. Moreover, the observed $B$-em-Cs have a variety of MM states, including $d$-type $\mathrm{MMs}, \mathrm{em}^{\mathrm{M}}$ and $\mathrm{em}^{\mathrm{M}}$ Cs, which is unexpected and very important accounting for the limited experimental data on MMs (mainly for the p-type-states) in FQHE [25], one-dimensional hybrid superconductor topological structures $[39,40]$ and in topological superconductor [41] . Thus, our observations give high impact to the physics of anyon/Majorana states and to the perspectives of their use for TQC. Note, that from five $\mathrm{em}$-Cs observed there is only one MMfree, which is a $12,1^{+}=5 / 2$ composite in the D07m dot, and, thus, MMs seen are intrinsic features of the confined electrons for $\gg 1$, in contrast to $k 1$ molecular states [16]. In the 
context of $\mathrm{em}^{\mathrm{M}}$ and $\mathrm{em}^{\mathrm{M}}-\mathrm{Cs}$, a SC analog of such states, which is called a spontaneous vortex phase, has been reported in the compound $\mathrm{EuFe}_{2}\left(\mathrm{As}_{1-x} \mathrm{P}_{\mathrm{x}}\right)_{2}$ [42].

The $\Delta_{v}$ steps observed demonstrate detection of fractional charge variations related to MMs using PL spectra. Moreover, the observed $\mathrm{CHs}$ are related to the states with and without MM, i.e., to a MM qubit (see Appendix D), and for the D07m and D09m dots these are zero-field qubits. This two-level qubit can be used in conventional schemes of quantum computing and/or quantized information bit of classical Boolean logics [43]. The data show that the appearance of the $\Delta_{\nu}$ steps depends on $D, N$ and $B$, or more generally on $N_{V}$ and $r_{s}$, and is suppressed with $r_{s}$ decrease. This gives the opportunity for engineering of $\mathrm{MM}$ qubits. The $\mathrm{CH}$ is related to the suppression of $a V$ generation under a decrease of the field and evidently reflects some fundamental interaction between $\mathrm{em} \mathrm{Vs}$ and a sign of the magnetic field variation, which needs further investigation.

\subsection{Magneto-electrons and fractional quantum Hall effect states}

Our experimental results presented here and in the previous studies $[15,16]$ show that the fractionally charged em-anyons emerge in the quantum confined (gapped) $e$ states, which allows superconducting-type sub-e PCLs/Vs generated by a single or a pair of es, creating ems. This allows consider a description of the FQHE in terms of a liquid crystal (LC), rather than as an incompressible liquid, in which specific $v_{n s}{ }^{+}-B-e m-C$ is a unit cell of the crystal. The nucleation of the $\mathrm{vns}^{+}-\mathrm{B}-\mathrm{em}-\mathrm{C} \mathrm{LC}$ is provided by a quasi-ordering of potential fluctuations (PF) [44] of the corresponding size as was suggested by us in [15]. The experimental evidence of such quasi-ordering is the observation of the Wigner-crystal-type network patterns of the localized electron states for $v=1$, observed in a scanning electrometer probe experiment in quantum Hall 2D-e structures [45].

Within the LC description, the conductivity plateaus are quantum-confined-type $L^{s}$ plateaus (see Figures B1b and B1d) rather than a set of states above the mobility edge of the disorder-broadened LLs. The $L^{S}$ plateaus strongly overlap, as follows from the $E_{L}{ }^{S}$ curves in Figure B1e, which is consistent with the models describing the temperaturedependence of the width of IQHE and FQHE plateaus [46], revealing a Lorentzian broadening of LLs. Moreover, LC description does not involve e-e interaction as shown in Appendix $B$.

We can suggest that the $\mathrm{em}^{\mathrm{M}}$ Majorana anyons revealed are the CFs of the quasi-particle description of the FQHE and they naturally explain the zero internal field of CFs, leading to a termination of the conductance plateaus at even fractional filling factors. The description of CFs using LC implies B-em-C consisting of $e^{1 / 20}$ and $e^{1 / 2-1}(0) \mathrm{ems}$, in which the latter contribute to the conductivity. The $S$-em-C of such type can explain the $1 / 2$ and $1 / 4$ fractional quantization of the conductivity of the holes and electrons in Ge [47] and GaAs [48] nanowires, respectively, in a zero magnetic field and the " 0.25 anomaly" of the conductivity of a point quantum contact $[49,50]$.

The signatures of $\mathrm{em}^{\mathrm{M}} \mathrm{S}$ in these experiments using confined geometries, i.e., probing of submicron areas, imply that the corresponding PFs are naturally formed in 2D-e structures and that their short- versus long-range order arrangement determine the formation the corresponding LCs and FQHE conductivity plateaus. Thus, in the extremely pure, high mobility structures in which FQHE plateaus emerge, the imperfection density is in a dilute regime $(\sim 1 \%)$, which provides a wide spectrum and long-range order of the PFs [44], induced by $S$ - and B-em-Cs. The PFs depth in 2D-e-structures is $\sim 10 \mathrm{meV}$ [51], which is an order of magnitude smaller than in $\mathrm{InP} / \mathrm{GaInP}_{2} \mathrm{QDs}$, and we can expect that the FQHE LC states will include $e m$-Cs having size $\sim 50 \mathrm{~nm}$, adopting only $s$ - and $p$-ems, and assume extended imperfections having size at least few $\mathrm{nm}$.

\subsection{Majorana anyons and topological quantum computing}

The proposal of the topologically protected qubit involving the $v=5 / 2$ state [31] is based on Laughlin's quasi-particle description of FQHE states [14], in which this state is assigned to a non-Abelian topological phase characterized by a Pfaffian wave function $[52,53]$. This state is considered as a set of degenerate $p$-wave paired fermions having 
charge $e_{0} / 4$. The qubit is formed by a zero-energy Majorana excitation of a fused fermion having charge $e_{0} / 2$. We can suggest that the $e m$ analog of the $e_{0} / 4$ fermion is $e^{2} 0_{0}+e^{1 / 8}{ }_{-1}(2) B$ $e m-C$, the $e 0 / 2$ state is $e^{2} 0+e^{1 / 8}-1(2)+e^{1 / 8}(2) B-e m-C$, formed by addition of two $e s$, and MM is $e^{2} 0+e^{1 / 8} 8_{-1}(2)+e^{1 / 8} 1(0) B-e m-C$. It follows from our results that it may be possible to realize and measure such $B$-em-Cs in $\mathrm{InP} / \mathrm{GaInP}_{2}$ using appropriate $N$ and $D$, and also to check the formation of the corresponding LC states in 2D-e structures using high-spatial-resolution optical [16] and electrometrical [54] measurements. Also, it may be possible to realize similar states using S-ems, thus creating magnetic-field-free TQCs.

\section{Conclusions}

We have used the magneto-PL spectra of $\mathrm{InP} / \mathrm{GaInP}_{2}$ QDs having seven electrons and a Wigner-Seitz radius $\sim 1.3$ to demonstrate self-formation of fractionally charged anion(magneto-electron) composites having $\sim \sim 5 / 2$ and Majorana modes/anyons. We observed the destruction of these composites and the appearance of anti-em composites induced by a magnetic field. Using a theoretical description of magneto-PL spectra and an analysis of the electronic structure of these dots based on single-particle Fock-Darwin spectrum and many-particle configuration interaction calculations, we show the formation of fractionally charged anion states based on the mechanism in which non-interacting quantum confined electrons generate sub-electron persistent current loops (vortexes). This implies the liquid-crystal nature of the fractional quantum Hall effect states, in which Majorana anyons are spontaneously formed, and opens new perspectives for the realization of topological quantum computing.

Author Contributions: A.M.M., D.V.L and A.S.V. conceived the magneto-PL NSOM experiment. S.B. preformed the configuration interaction calculations. The manuscript was written by A.M.M with critical input from all authors. All authors have read and agreed to the published version of the manuscript."

Funding: A.M.M and A.S.V acknowledge support from the Russian Science Foundation grant No 19-19-00246; a portion of work related to initial selection of quantum dots using cryo-NSOM measurement was supported the National Science Foundation grant \#DMR-1904610.

Data Availability Statement: All data needed to evaluate the conclusions in the paper are present in the paper

Conflicts of Interest: All authors declare that they have no competing interests.

\section{Appendix A. Theory of photo-luminescence specra of quabtum Hall pudlles}

\section{A.1 General model}

The PL spectrum of a QD having $N$ electrons arises from the radiative recombination of the photo-excited hole $(h)$ state in the valence band with $N^{*}=N+1$ es in the conduction band, which together form the photo-excited state (PS) of the QD. This PS is formed after absorption of the incoming photon by QD in initial state (IS) creating an $e-h$ pair, as shown in Figure A1a. Accounting for the fact that IS can have fractionally charged ems, we assume that all the interactions leading to formation of such a state are included in the formation of the $e-h$ pair and PS state and do not affect photo-excitation and radiative recombination processes. This implies that for an IS having ems the generated and recombined $e-h$ states have fractional charge.

The energy of the photon of the exciting laser is $\hbar \omega_{\mathrm{ph}} \sim E_{0}+E_{\mathrm{gr}} \mathrm{QD}+\Delta E_{\text {exc, }}$ where $E_{0}$ is a band gap energy of the material, $E_{\mathrm{gr}} \mathrm{QD}$ is the ground state energy of $N^{*} e-h \mathrm{QD}$, and $E_{\text {exc }}$ is 


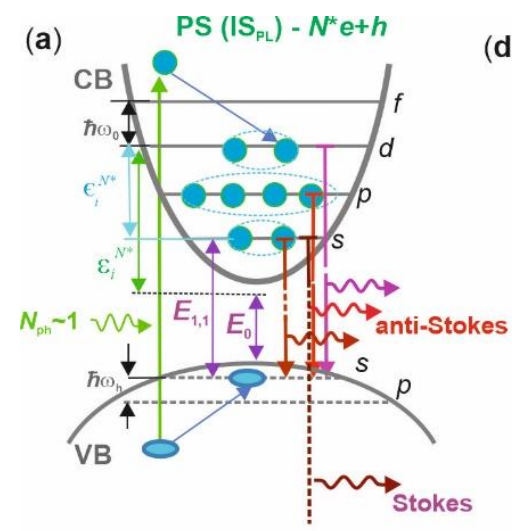

(d)

(b)
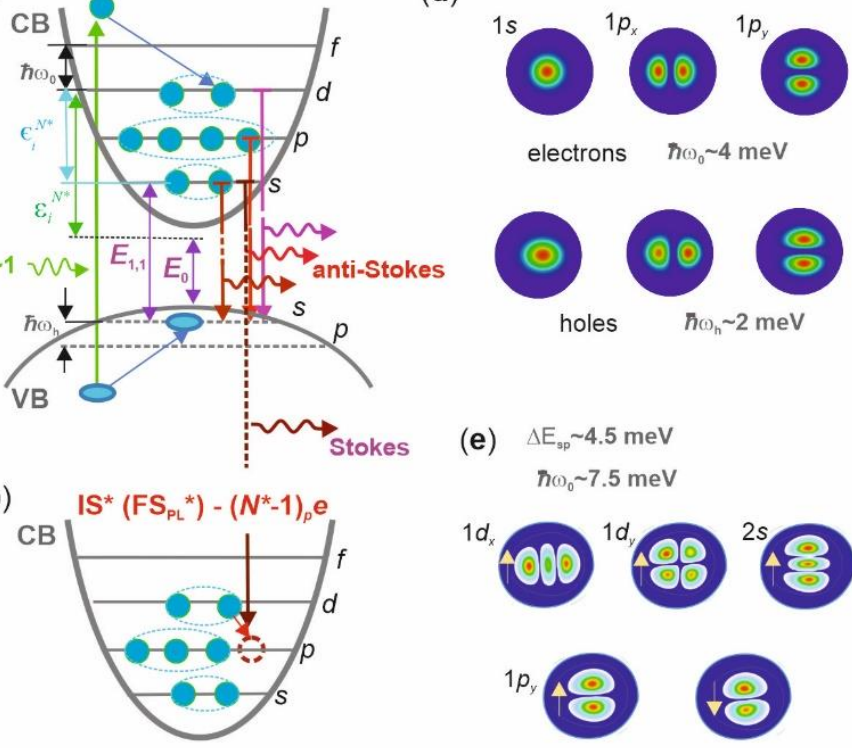

electrons $\hbar_{\omega_{0}} \sim 4 \mathrm{meV}$

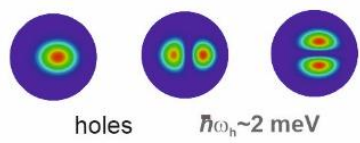

(e) $\Delta \mathrm{E}_{\mathrm{sp}} \sim 4.5 \mathrm{meV}$

$\hbar \omega_{0} \sim 7.5 \mathrm{meV}$

(c)
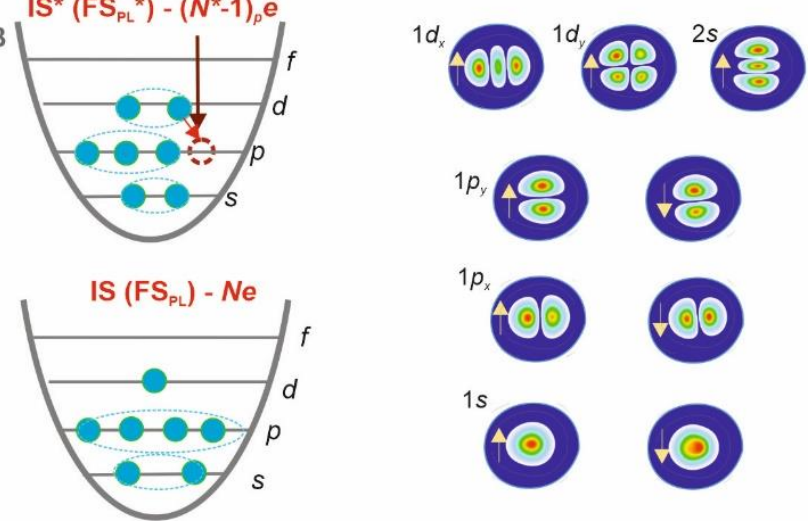

Figure A1. (a) Population of electrons (solid circles) and hole (solid ellipse) in the $s, p$ and $d$ PS levels in conduction (CB) and valence (VB) bands of $\mathrm{InP} / \mathrm{GaInP} \mathrm{P}_{2} \mathrm{QD}$ in PL experiment under low photon pumping $\left(N_{\mathrm{ph}} \sim 1\right)$. Parabolas with horizontal lines inside are $\mathrm{CB}$ and $\mathrm{VB}$ potential of QD with quantum confined $e$ and $h$ levels, thick vertical up(down)/snaked arrows are excitation(emission)/photons, inclined arrows show $e-h$ relaxation, thin vertical arrows are electron energies involved in PL transitions (see Equation A3).

$(\mathbf{b}, \mathbf{c})$ Population of electrons/hole (solid/empty circles) in CB of IS* $\left|v_{f *}^{k}\right\rangle$ (b) and IS (c) states.

(d) Probability densities (size 200×200 $\mathrm{nm}^{2}$ ) of the three lowest electron (upper) and hole (lower) states of InP/GaInP2 QD $(D \sim 100 \mathrm{~nm})$ calculated using $8 \quad \bar{k} \cdot \bar{p}$ band model.

(c) HF electron density distributions (size 100x100 $\mathrm{nm}^{2}$ ) of the six occupied states having total spin $S_{z}=3 / 2$ of $\mathrm{InP} / \mathrm{GaInP} \mathrm{P}_{2}$ QD having $N=9$ and $\hbar \omega_{0}=7.5 \mathrm{meV}(D \sim 100 \mathrm{~nm})$. Arrows show spin direction.

the excess excitation energy $(\sim 500 \mathrm{meV})$. After photon absorption, the photo-excited $e-h$ pair relaxes (loosing Eexc by phonon emission) to the ground PS state, which is the initial state of the PL process (ISpL). The many-particle Hamiltonian of the PS-ISpL state can be written, using composite indexes $i$ for a single particle state $|i\rangle$ and accounting for the Coulomb interaction [55], as

$$
\begin{array}{r}
H^{P S}=\sum_{i} \varepsilon_{i}^{e} c_{i}^{\dagger} c_{i}+\sum_{n} \varepsilon_{n}^{h} h_{n}^{\dagger} h_{n}+\frac{1}{2} \sum_{i j k l}\left\langle i j\left|V_{e e}\right| k l\right\rangle c_{i}^{\dagger} c_{j}^{\dagger} c_{l} c_{k} \\
+\sum_{i n m j}\left\langle i n\left|V_{e h}\right| j m\right\rangle c_{i}^{\dagger} h_{n}^{\dagger} h_{m} c_{j} .
\end{array}
$$

The operators $c_{i}$ and $c_{i}^{\dagger}\left(h_{n}\right.$ and $\left.h_{m}^{\dagger}\right)$ create and annihilate an electron (a hole) in a single particle (SP) state $|i\rangle(|n\rangle)$, where there are $N^{*}$ electrons. The first two terms in Equation A1 are the kinetic energy of the electrons and the hole, quantized by $\hbar \omega_{0}$ and $\hbar \omega_{h}$, respectively, where $\omega_{0}$ and $\omega_{h}$ are the harmonic frequencies of $e$ and $h$ in the parabolic confinement potential. The third and the fourth terms are scattering terms described by corresponding two-body matrix elements of $e-e[56,57]$ and $e-h[57,58]$ Coulomb interactions, respectively. 
For the final PL state (FSp), which is the IS of the QD (see Figure A1c), the Hamiltonian $H^{I S}$ does not have the fourth term of Equation $\mathrm{A} 1$ and there are $N$ electrons and no hole. The PL spectrum, i.e., the dependence of the emission intensity on the photon frequency $\operatorname{IPL}(\omega)$, is determined by Fermi's Golden Rule:

$$
I_{P L}(\omega)=\frac{2 \pi}{\hbar} \sum_{f}\left[\left\langle v_{f}|\mathcal{P}| v_{i}\right\rangle\right]^{2} \gamma_{f}^{L}\left(\varepsilon_{i}-\varepsilon_{f}-\hbar \omega\right),
$$

where $\left|v_{i}\right\rangle$ and $\left|v_{f}\right\rangle$ are the initial and final states of the QD having corresponding energies $\mathcal{E}_{i}$ and $\varepsilon_{f}$. The inter-band polarization operator $\mathcal{P}=\sum_{k n}\langle k \mid n\rangle c_{k} h_{n}$ removes an $e-h$ pair from the PS state $\left|v_{i}\right\rangle$. The matrix element (overlap integral) $\langle k \mid n\rangle$ determines the selection rules. Since there is only a single valence band hole we can define a purely electronic PS state $\left|v_{i}^{k}\right\rangle=\sum_{n}\langle k \mid n\rangle h_{n}\left|v_{i}\right\rangle$ [55]. The operator $\mathcal{P}$ create a hole in PS $\mathcal{P}\left|v_{i}\right\rangle=$ $\sum_{k} c_{k}\left|v_{i}^{k}\right\rangle$ and leaves the QD in the excited state $\mathrm{IS}^{*}$-FS $\mathrm{PL}^{*}$ (see Figure A1b), having energy $\check{\epsilon}_{k}^{N}$.This state can be degenerate with a few other IS* configurations, which can be mixed by Coulomb exchange leading to a splitting of the PL transition [55]. Such a contribution is important in relatively small excitonic QDs [59], which are not considered here.

The contribution of the IS* state to the PL spectrum is described by a difference $\left|\Delta^{k}\right\rangle=\left|v_{f *}^{k}\right\rangle-\left|v_{f}^{k}\right\rangle$ and it is important in dots having a molecular electron arrangement, i.e., $r_{\mathrm{s}}>2[16]$.

Accounting for a low temperature $\left(\hbar \omega_{0}, \hbar \omega_{h}<k T\right)$ and low pumping rate $\left(N_{\mathrm{ph}} \sim 1\right)$, generating only one $e-h$ pair in the dot in IS ${ }^{\mathrm{PL}}$ (see Figure A1a), the PL spectrum, can be written as:

$$
\begin{aligned}
& I_{P L}(\omega)=\langle 1 \mid 1\rangle^{2} \gamma_{1}\left(E_{1,1}-\hbar \omega\right)+\sum_{i \neq 1}\langle i \mid 1\rangle^{2} \gamma_{i}\left(E_{1,1}+\epsilon_{i}^{N *}-\hbar \omega\right) \\
& -\sum_{k}\left\langle\Delta^{k} \mid \Delta^{k}\right\rangle^{2} \gamma_{k}\left(E_{1,1}-\check{\epsilon}_{k}^{N}-\hbar \omega\right),
\end{aligned}
$$

where $E_{1,1}=E_{0}+\varepsilon_{1}^{N *}-\varepsilon_{1}^{N}+\hbar \omega_{h}, \quad \varepsilon_{i}^{N *}\left(\varepsilon_{i}^{N}\right)$ is the many-particle orbital energy of $i$ electron in PS(IS), $\epsilon_{i}^{N *}=\varepsilon_{i}^{N *}-\varepsilon_{1}^{N *}$ is difference in the kinetic (confinement) energy between the higher and the lowest states, $\breve{\epsilon}_{k}^{N}$ is the energy of the $\left|v_{f *}^{k}\right\rangle$ state and $\gamma_{i}(\omega)$ is a Lorentzian spectral function of the corresponding transition, describing the final width of the spectral lines and replacing of the delta function used in Equation A2.

The PL spectrum thus has three contributions: a "zero" energy s-line (first term) accompanied by the anti-Stokes (ASCs) and Stokes (SCs) components (second and third terms, respectively). The SCs arise from the "molecular" contribution to the electron distribution, i.e. center-of-mass and relative electron vibrational modes and, in general, include combinations of translational, breathing and rotational modes, having frequencies $\frac{\breve{\epsilon}_{k}^{N}}{\hbar} \sim \omega_{0}, \sim 1.8 \omega_{0}$ and $\sim 0.2 \omega_{0}$, respectively [33].

\section{A.2 Single-particle states of confined electrons}

To describe the SP states (SPSs), we have used the FD spectrum $E_{k l}(B)=E_{k, l} \mathrm{FD}(\Omega)$, where $\Omega^{2}=\omega_{0}^{2}+\omega_{c}^{2} / 4, \omega_{c}=e^{*} B / m^{*}$ is a cyclotron frequency, $k=0,1, \ldots$, is a principal quantum number, $l=k-|m|$ is a radial quantum number and $m=-k,-k+2, \ldots, k-2$ is an azimuthal quantum number [60]. Circular symmetric FD states (FDSs) 00-, 01, 10-, 02-, 11-, 20- and so on correspond to 2D atomic-like states $1 s^{-}, 1 p_{x^{-}}, 1 p_{y^{-}}, 1 d_{x^{-}}, 1 d_{y^{-}}, 2 s^{-}$and so on.

We also analyzed SPSs accounting for the atomic crystal structure of InP/GaInP2 ${ }_{2}$ dots using the 8-band $\mathbf{k} \cdot \mathbf{p}$ method [36]. In Figure A1d we show the probability density distributions of the $1 s, 1 p_{x}$, and $1 p_{y}$ states calculated for a circular dot having size $100 \mathrm{~nm}$. The calculations give $\hbar \omega_{0}=4 \mathrm{meV}$ and $\hbar \omega_{h}=2 \mathrm{meV}$. They show that the spatial extent of $e$ and $h$ states in these QDs is nearly the same (despite a two-times difference in the confinement energy) and that their wave-functions show FD type $s$ - and $p$-shell spatial structure. For the $h$, however, the spatial extent is two times larger than that of FD states, which is a result of an inter-valence-band mixing induced by a piezo-electric field. The piezo-electric field also leads to a $\sim 10 \mathrm{~nm}$ separation of the $e$ and $h$ along the $x$-direction, which is [-110]. This makes transitions involving $x$-type electron shells allowed. 


\section{A.3 Effect of Coulomb interaction on confined electrons states}

The $e-h$ Coulomb interaction between the $N^{*} e$ and single $h$ (the fourth term in Equation A1) mixes single-band $h$ states (intra-band mixing) allowing PL transitions for all es in QD [34] as shown in Figure A1a. Accounting for the mixing we can approximate the distribution of the density of the $h$ in the lowest state by a nearly uniform positive charge distribution having weak modulations induced by spatial variations of the occupied electron shells and extending over the whole QD size. Thus, we can neglect the effect of the $e-h$ interaction on $\varepsilon_{i}^{N *}$ and include only e-e interaction in analysis of PS.

In Figure A1e we present the electron density distributions (EDDs) of the occupied PS shells of a QD having $N=9, \hbar \omega_{0}=7.5 \mathrm{meV}(D \sim 75 \mathrm{~nm})$, total spin $S_{z}=3 / 2$ and a non-circular dot shape (analog of D07m) calculated by the Hartree-Fock (HF) method. The densities have minor shape deviations from that of the single-particle ones in Figure A1d, except $(1 d-2 s) x$ shell hybridization together with slight asymmetry of the states having different spin (see $s$-state) and spatially split parts for non-zero angular momentum states (see spin down $p_{x}$-state), which appear from the non-circular shape. The $e-e$ interaction, however, gives a renormalization of the shell splitting, resulting in $\varepsilon_{i}^{N *} / \varepsilon_{i}^{e} \sim 0.7$. This shows that the electron states in InP/GaInP2 2 Ds can be approximated by FDSs having screened $\hbar \omega_{0}^{*}=\varepsilon_{i}^{N *} \approx \Delta E_{s p} \approx \Delta E_{s d} / 2$, where $\Delta E_{\text {sp }}$ and $\Delta E_{s d}$ is s-p and s-d splitting.

\section{A.4 Magnetic field dispersion of photoluminescence spectra}

The shift of $s$-line in magnetic field is

$$
E_{\mathrm{s}}(B)=E_{1,1}(B)-E_{0}=\varepsilon_{1}^{N *}(B)-\varepsilon_{1}^{N}(B)+\hbar \omega_{h}(B),
$$

where for the parabolic potential, for which the energy of center-of-mass motion $\hbar \omega_{0}$ does not depend on $N[60]$, the Coulomb energy term is

$$
\varepsilon_{1}^{N *}(B)-\varepsilon_{1}^{N}(\mathrm{~B})=\Delta E_{s}^{C}(B)=\sum_{N} V_{1 i, e e}^{N *}(B)-\sum_{N-1} V_{1 i, e e}^{N}(B),
$$

and depends on the difference between the $e-e$ interaction energy of the $s$ electron in the PS and IS. The matrix elements in Equation A4b can be written as $V_{1 i, e e}^{N *}=V_{e e}(B) \approx$ $\sqrt{\frac{\pi}{2}} \frac{e^{* 2}}{\varepsilon} \frac{1}{l_{B}}$, where $l_{B}^{2}=\hbar c /\left(e B \sqrt{1+\frac{4 \omega_{0}^{2}}{\omega_{c}^{2}}}\right)$ [56]. At zero field, $\Delta E_{s}^{C}(0) \approx r_{s} \cdot k \cdot \hbar \omega$, where $k$ is the level number ( 1 for $1 s-, 2$ for $1 p$-, 3 for $1 d$ - and $2 s$-states and so on) of the photo-excited $e$. For a pure electron state (no ems), $\Delta E_{s}^{C} \approx N^{*} \cdot V_{e e}(B)$ and

$$
E_{\mathrm{s}}(B) \approx N^{*} \cdot V_{e e}(B)+\hbar \omega_{h}(B)
$$

The formation of $e^{v}$ ems corresponds to a reduction of the charge from $e_{0}$ to $e_{0} / v$ and results in a decrease of $V_{e e}(B)$. Since $N^{*} \cdot V_{e e}(B)<\hbar \omega_{h}(B)$, the effect on $E_{\mathrm{s}}(B)$ is expected to be weak.

For the ASCs the $B$-shift is

$$
\epsilon_{i=k, l}^{N *}(B)=E_{k l}(B)+E_{S}(B)
$$

and the experimental value $\epsilon_{i=k, l}^{N *}(B)-E_{S}(B)$ directly measures the corresponding FDSs dispersion. The same is valid for SCs. In Equation A5 the B-dispersion of $E_{k l}(B)$ term directly depends on $\omega_{\mathrm{c}}$ and the values of $v$ can be measured using the fit of the corresponding FDSs in the form $E_{k l}(B)=E_{k l}\left(B_{e}+B_{v}\right)$, where $B_{\bullet}$ accounts for internal field generated by $V$ s to experimental data, as was shown by us for dots having $r_{s}>2$ and $k 1$ in Refs. 15, 16. 


\section{Appendix B. Ground states of quantum Hall puddles}

\section{B.1 Total angular momentum plateaus of confined electrons in magnetic field}

In Figures B1a and B1b we show the total energy $E^{S_{\text {tot }}}$ and total angular momentum $L_{z}$, respectively, versus $B$ calculated using $C I$ for $N=8$ and $\hbar \omega_{0}=5.8 \mathrm{meV}$, corresponding to the PS of the QD D09m. We used reduced units $v s^{-1}=L^{s} / L_{0}=-L_{z} s_{z} / L_{0}$, where $L_{0}=N(N-1) / 2$ $[16,61]$ and $v^{-1}=B / B_{0}$, where $B_{0}$ is a field corresponding to $v=1$, and $B\left(v^{-1}\right)$ ranges up to $\sim B_{0}(1)$. The $v S^{-1}\left(V B^{-1}\right)$ curves are presented for all total spin $S_{z}=S$ states from 0 to 4 and for $S>1$ the curves are truncated for $\mathcal{B}^{-1}>0.25$. The inset in Figure B1a shows $E^{S_{\text {tot }}}$ for $N=7$ and $S$ from $1 / 2$ to $5 / 2$ related to the IS of the QD D09m. The upper inserts in Figure B1b show the calculated zero field CDDs for $N=8$ and 7 , and the lower ones show the HF EDD of the 10- $p_{x}$ state with a few $V$ circles adjacent to each other, which represent a tentative description of the $e m$ structure. $E^{S_{\text {tot }}}$ and $L_{z}$ for $N=6$ (the IS of D01m) were calculated in Ref. 16.

The CDDs give $D \approx 85 \mathrm{~nm}$ and $r \approx \approx 1.5$ (see Table 1). They have two minima having size $\sim 15 \mathrm{~nm}$ and separated by $\sim 30 \mathrm{~nm}$ along the $x$-axis, which can be assigned to $(7,1)$ and $(6,1)$ isomers in a molecular arrangement in the limit of $r_{\mathrm{s}} \gg 1$ [62], for $N=8$ and 7, respectively. For $N=7$, the CDD show enhanced density (clustering of electrons) along the $y$ direction.

The $E^{S_{\text {tot }}}\left(v^{-1}\right)$ energies in Figure B1a and in the insert are nearly constant up to $V^{-}{ }^{-}$ $1 \sim 0.6$ and then increase linearly with $B$ increase. The former corresponds to weak fields $\left(\omega_{c}<\omega_{0}\right)$, for which the kinetic and Coulomb energy, related to quantum confinement, $E_{\text {conf }}=16 \hbar \omega_{0}{ }^{*}$, and center-of-mass motion, $E_{\mathrm{c} . \mathrm{m}} \approx r_{\mathrm{s}} E_{\mathrm{conf}}$ are dominant; the latter corresponds to strong fields $\left(\omega_{c}>\omega_{0}\right.$, $)$ when the cyclotron energy of electrons, $E_{\mathrm{cyc}}=4 \hbar \omega_{\mathrm{c}}$, becomes dominant [16].

As can be seen from the comparison of $E^{S_{\text {tot }}}\left(V^{-1}\right)$ curves, the boundary between weak and strong fields is $S$ dependent and increases from $v^{-1} \sim 0.5$ for $S=0$ to $v^{-1} \sim 1$ for $S=4$.

The analysis of the curves also give $B_{0}=7.2 \mathrm{~T}$, which is a field at a crossing point between the $E^{3}{ }_{\text {tot }}\left(\nu B^{-1}\right)$ and $E^{4}{ }_{\operatorname{tot}}\left(\nu B^{-1}\right)$ curves. This value is equal to $B_{0}=A \cdot(h / e)\left(4 N / \pi D^{2}\right)$, where a geometrical factor $A=1.25$.

The $E^{S_{\text {tot }}\left(\nu B^{-1}\right)}$ curves also have weak modulations, having amplitude $\leq 0.5 \mathrm{meV}$ and period $\Delta \mathrm{vB}^{-1} \sim 0.2$, which are considered to arise from the energy of relative electron motion $E_{\text {rel }}[16,61]$. A set of minima of the modulations at $v=8$ and $7 / 3$ for $S=0$ and at $v=7 / 2$ and 2 for $S=1$ for $v^{-1}<0.6$ correspond to a set of GSs $\mid v_{n s}>N_{1 / v B}$, where $n$ numbers the GSs in the set, which is $\mid 8_{0}, 7 / 21,7 / 3_{0}, 2_{1}>8_{0.6}$. For $N=7$ the set is $\mid 9 / 21 / 2,5 / 2_{1 / 2}>7_{0.6}$, for $N=6$ it is $131 / 2,5 / 3_{1 / 2}>6_{0.6}$ and the GS sets are different for different $N$. Note, that the difference between $E_{\text {tot }}(0)$ values, $\Delta E_{\text {tot }}=36 \mathrm{meV}$ for $N=8$ and 7 , which corresponds to $\Delta E_{s}^{C}(0)=\Delta E_{\text {tot }}-3 \hbar \omega_{0}=18 \mathrm{meV} \approx r_{s} \cdot k \cdot \hbar \omega_{0}$ (see below C.1).

In Figure B1b the $v s^{-1}\left(v_{B}\right)^{-1}$ curves reveal $L^{S}$ plateaus. The plateaus follow energy modulations and stay between the modulation maxima, having, thus, the same period (width). Their crossing with $v s^{-1}=v_{B}^{-1}$ line give the values of $v_{n}$ as shown in Figure B1b for both $N=8$ and 7 . The plateaus recall the same of the transversal conductance of IQHE and FQHE [63], which gives evidence on the critical role of localization, i.e., quantum confinement, in the formation of IQHE and FQHE states.

\section{B.2 Single-particle Fock-Darwin ground states and vortexes}

In Figure B1c we present the FD spectrum $E_{k, l}\left(v^{-1}\right) / \hbar \omega_{0}\left(\hbar \omega_{0}=\hbar \omega_{0}^{*}=3.5 \mathrm{meV}\right)$, which is related to D09m, plotted up to $3 \hbar \omega_{0}$ and $v^{-1}=1.15$, which includes levels up to 13 and 07 . Using this spectrum, we calculated the total angular momentum $L \mathrm{FD}^{S}$ and energies of the corresponding states $E_{L}{ }^{S}$ versus $v^{-1}$ for $N=8$ and $S=1$, which are shown in Figures B1d and B1e, respectively. For the calculation of the $E_{L} S$ energy, we use the expression $E_{L} S=16 \cdot \hbar \omega_{0} \cdot r_{s}+\sum_{N} E_{k l} L^{L(S)}$, where the first term is the Coulomb energy and $E_{k l} L^{(S)}$ are FDSs constituents of the $L(S)$ state. In Figure B1d, we also show $L F D, v^{1}=L v+L \mathrm{FD}^{1}$ (see Appendix C.1), which is also shown in Figure B2b. 


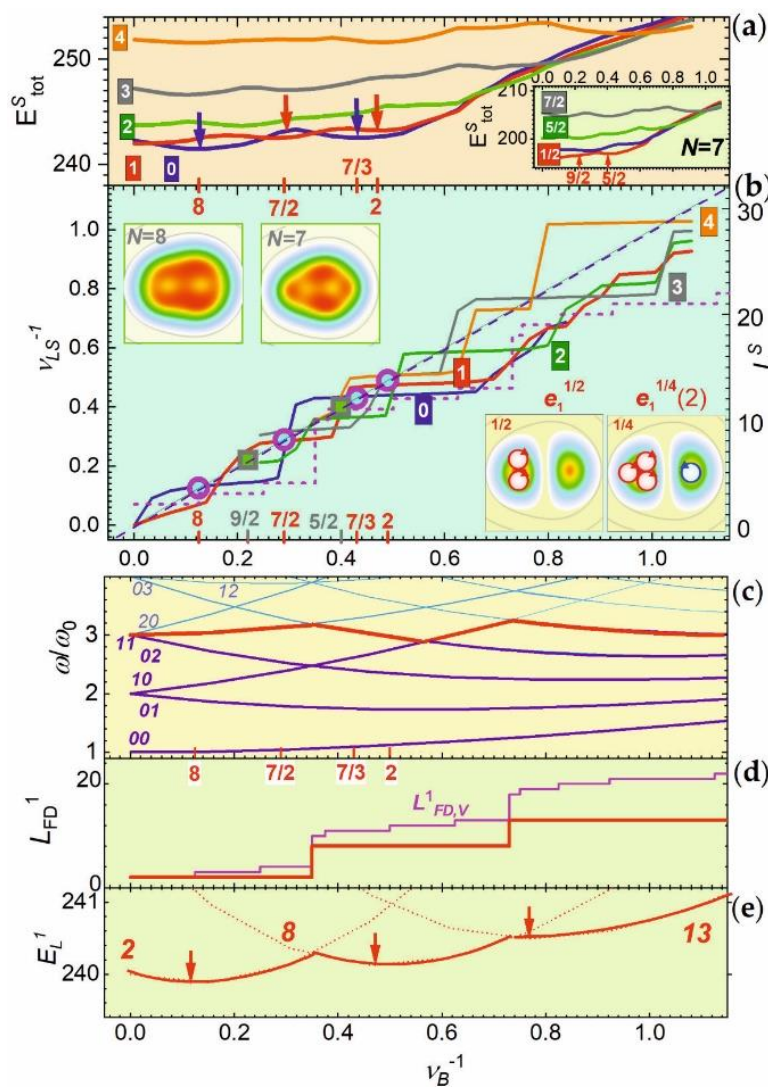

Figure B1. Total energy $E^{S_{\text {tot }}}(\mathbf{a})$ and reduced total angular momentum $u s^{-1}=L s / L_{0}(\mathbf{b})$ for different $S$ (solid curves marked by numbers) versus reduced field $v^{-1}$ for $\hbar \omega_{0}=5.8 \mathrm{meV}$ and $N=8$ calculated by CI method. Insert in (a) presents $E^{S}$ tot for $N=7$. Vertical arrows in (a) mark GSs (see composition numbers on $V^{-1}$ axes). The dashed line in $(\mathbf{b})$ is the $v^{-1}=V^{-1}$ dependence, circles(squires) mark GSs for $N=8(7)$, dashed curve is the $L F D, v^{1}$ function (see also (d). The inserts show calculated total CCD (upper) for at $N=8$ (left) and $N=7$ (right) at $B=0 \mathrm{~T}$ and HF EDD of $1 p_{x}$ single particle state (lower) with $V \mathrm{~s}$ (circles with arrow, showing circulation direction), corresponding to $e^{1 / 2} \mathrm{em}$ (left) and its MM (right). The CCD and EDD size is $100 \times 100 \mathrm{~nm}^{2}$.

FD spectrum (dark and light color curves are occupied and unoccupied levels, respectively) $E_{k, l} \mathrm{I}^{\mathrm{D}}\left(v_{B^{-1}}\right) / \hbar \omega_{0}^{*}$ calculated for $\hbar \omega_{0}^{*}=3.5 \mathrm{meV}$ (c) together with total angular momentum (thick curve) $\operatorname{LFD}^{1}\left(V^{-1}\right)\left(\right.$ d) and energies (thin dot curves) $E_{L}{ }^{1}\left(V^{-1}\right)$ of $N=8$ and $S=1$ (see thick lines outlining topmost levels in (c)) (e). Thick solid lines in (e) outline minima of $E_{L}{ }^{1}\left(V^{-1}\right)$ and vertical arrows mark corresponding GSs. Thin line in (d) is $L F D, V^{1}$ function.

The topmost $S=1$ FDSs in Figure B1d show three crossings at $v^{-1} \sim 0.35,0.57$ and 0.74 . The last crossing corresponds to the transition between the weak and strong field of Figure B1a, in which all electrons occupy the lowest Landau level. As can be found from an analysis of the FD spectrum in Figure B1c, this crossing shifts to larger fields with step $\Delta v^{-}$ $1 \sim 0.1$ when $S$ increases, which is in good agreement with the $E^{S_{\text {tot }}}$ crossings and, thus, allows one to explain this shift using a single-particle description.

Between the crossings, a set $\left\{l_{z}\right\}_{N}$ is conserved resulting in the $L_{\mathrm{FD}}{ }^{S}$ plateaus (see Figure B1d). Within a specific $L_{F D} S$-plateau, the energy of the corresponding state $E_{L} S$ is the lowest and has a minimum, which gives modulations of the total energy and a set of GSs (see Figure B1e), like that in Figure B1a. Thus, the plateaus and discrete GSs of electrons in magnetic field arise in QDs due to quantum confinement and they appear without accounting for the $e$-e interaction. The $\operatorname{LFD}^{S}$ plateaus, however, have one-and-a-half-time larger period and two times smaller amplitudes than $L^{S}$ ones, which implies contribution of additional non-zero $l_{z}$ states, missing in FD spectrum. It is naturally assume that these states are the $V \mathrm{~s}$, which add $l_{z}=1$ each. Since at $v=1$ the number of $V \mathrm{~s} N_{v}=N^{*}$, the contribution of $V \mathrm{~s}$ to $L^{S}$ can be approximated by a stepped function $L v$, in which $l_{z}=1$ step is added at $v^{-1} \sim k / N^{*}$, where $k$ is integer. A $L F D, V^{1}$ function, accounting for their contribution $\left(L F D, V^{S}=\right.$ 
$L \mathrm{FD}^{S}+L v$ ), has increased period amplitude (see Figure B1d) and approximately matches $L^{S}$ (see Figure B1b). Thus, accounting for Vs and single-particle states seems to be adequate for the description of the ems states.

\section{Appendix C. Fractionally charged ground states}

\section{C.1 Magneto-electron composites and Majorana modes}

The fractional $v_{n s}$ implies the formation of ems. Based on our previous studies [15, 16], we suggest that for the $p_{x}$ state and $v=1 / 2$ the $\mathrm{em}$ structure consists of two vortexes (Vs) generated at the center of the left half of the EDD at $x \sim-50 \mathrm{~nm}$, which corresponds to a clockwise phase change of the electron wave-function (see right lower insert in Figure B1b). We denote this $e m$ as $e_{1}^{1 / 2}$, where we add a subscript $l=l_{z}$ in the $e m$ notation, which further will be $e l^{v}$. For a paired $e$-state $e m$ is $e^{2 v}{ }_{l}$.

The adjacent right insert shows corresponding MM, i.e., $e^{1 / 4} \mathrm{em}$, in which single $V$ and anti- $V(a V)$ are added. The $a V$ is generated in the right half of $p_{x}$-state at $\mathrm{x} \sim+50 \mathrm{~nm}$, which has anticlockwise phase change of electron wave-function. The $e_{1}^{1 / 4} \mathrm{em}$ can also have a configuration consisting of two $V \mathrm{~s}$ and two $a V \mathrm{~s}$, which exist at zero $B$ and, thus, can be considered to be a Majorana magneto-electron/anyon $\left(\mathrm{em}^{\mathrm{M}}\right)$. To distinguish it from a MM, we add the number Vs of the "host" em to the MM-type ems notation. Using such a notation, one can see that $e_{1}^{1 / 4}(0)$ is $e m^{M}$ and $e 1^{1 / 4}(2)$ is a MM. Note that there exists $e 1^{1 / 2}(0) e m^{M}$, which implies that $e^{1 / 4}(0) \mathrm{em}$ is the MM of $e_{1}^{1 / 2}(0) \mathrm{em}^{\mathrm{M}}$.

For the $p_{y}$-state, the $V \mathrm{~s} / a V \mathrm{~s}$ generated are located along $y$ at distances $\pm 50 \mathrm{~nm}$ and the $V \mathrm{~s}$ of $e_{1^{1 / 2}}$ and $e-1^{1 / 2} \mathrm{ems}$ do not overlap. The same can be generally valid for other $l_{z}$ states and, thus, different $l_{z}$ states can form ems simultaneously and independently creating a multi-em state, i.e., an em-composite (em-C).

The classification of the em-Cs can be done by listing the values of $u$, which are the us of $e l^{v}$ ems, mapping of $V \mathrm{~s}$ positions as shown in the insert in Figure B1b and specification of the total composition value $v_{n}$, which we set up as

$$
v_{n S}=\left(w_{\mathrm{Nie}}+\mathrm{N}_{\mathrm{em}} / \mathrm{N}_{V}\right) \mathrm{s}
$$

where $v_{N i}$ is an integer value related to $N i$ electrons, $N_{e m}=N-N_{i}$ is the number of $e m s$ and $N_{V}=\sum(u)^{-1}$ is the total number of $\mathrm{em}^{\prime} \mathrm{s} V \mathrm{~s}$. For MM em-Cs having a number of $a V_{\mathrm{s}} N_{a V} \leq N_{V}$, there is an additional, effective composition value $v s^{+}$, which is

$$
v_{n S}{ }^{+}=\left({ }_{\text {Nie }}+N_{e m} / N v^{+}\right) S_{,}
$$

where $N_{V}{ }^{+}=N_{V}-2 N_{a V}$ is the number of uncompensating $V$ s determining $B_{v}$. The reduced charge of the $\mathrm{em}-\mathrm{C}$ is

$$
e_{N}{ }^{*}=e_{N} / e_{0}=\left(N_{i}+\sum n l u\right) / e_{0},
$$

where $n l$ is the SPS occupation equal to 1 or 2 .

A change of $v_{n}$ of the PS and IS $\mathrm{em}$-Cs at the boundaries of the $L^{s}$ plateaus can give $\Delta_{\nu}$ steps in the dispersion of the s-peak, which can be written as:

$$
\Delta_{\nu} \approx b \cdot \Delta E_{s}^{C}(0) \cdot\left[\left(\frac{\Delta e_{N}}{e_{N}}\right)_{2}-\left(\frac{\Delta e_{N}}{e_{N}}\right)_{1}\right],
$$

where $b$ is a coupling coefficient, assumed to be unity, and $\left(\frac{\Delta e_{N}}{e_{N}}\right)_{1,2}=\left(\Delta N_{i}+n_{l}^{p} v_{l}^{p}+\right.$ $\left.\sum \Delta n_{l} \Delta v_{l}\right)_{1,2}$ and the superscript $p$ denotes a photo-excited $e$, is a relative charge between PS and IS of adjacent $\mathrm{em}-\mathrm{C}_{1}$ and $\mathrm{em}-\mathrm{C}_{2}$ composites. The steps are positive(negative) when $N_{V}$ in IS(PS) of $\mathrm{em}-\mathrm{C}_{2}$ is larger than the same in $\mathrm{em}-\mathrm{C}_{1}$ and vice versa. In our dots $(N=7$, $k=3$ and $\hbar \omega_{0} \sim 6 \mathrm{meV}$ ), $\Delta E_{s}^{C}(0) \sim 20 \mathrm{meV}$ (as discussed above in B.1) and for single $e^{1 / 2} \mathrm{em}$, i.e., two $V_{\mathrm{s}} N_{V}$ difference, the expected step amplitude is $\Delta \sim 1 \mathrm{meV}$. The Equation A4c thus can be written as 


$$
E_{\mathrm{s}}{ }^{m-\mathrm{C}}(B) \approx N \cdot V_{e e}(B)+\hbar \omega_{h}(B)+\Delta_{v}
$$

\section{C.2 Fock-Darwin spectrum fit}

The ems composition of the single particle $e$-states were obtained using a fitting procedure. In the procedure we, first, extracted the experimental $p$ - and $d$-FD states values from the data in Figures $3 a-3 c$ using the subtraction of the $s$-shift from the $p$-and $d$-shifts, respectively; second, we fitted calculated FD energies to the experimental data by adjusting the cyclotron frequency $\omega c$, which give $n$ values; third, we plotted the experimental and calculated FD shifts, denoted them by $e^{v_{l}}$ (see Figures 4a-4c), together with $s$-shift and the calculated $\Delta_{v}$ and $E_{s}{ }^{m-m-C}$ values. The results are plotted using reduced units, like that in figure B1c. This allows a unified description of the e-em-FD spectrum of different dots. The fitted curves include an adjustment of the zero field $p$ - and $d$ peak positions for dots D07m and D09m.

The fit accounts for a decrease of the $B$-dispersion due to a $1 / v$-times decrease of $\omega c$, which in the limit $1 / v \rightarrow 0$ comes out to be dispersionless, i.e., a zero shift, eliminating the level crossing. Thus, for the states having negative $l_{z}$ (see the FD spectrum in Figure B1c) the ems lead to an increase, while for zero and positive $l_{z}$ to a decrease of the energy, towards a zero field value. For negative $l_{z}$ this can be traced for $e^{1 / 2-2}$ and $e^{1 / 4-2} B$-ems of D01m in Figure 4a.

\section{C.3 Construction of the em-C structure}

The $e m-C$ structure is represented by the $V$ s overlaid on the CDD of the dot. To plot it, we used the $u$ values obtained and put $(u)^{-1}$ arrowed circles of individual $V \mathrm{~s}$ in the region occupied by corresponding HF EDD (see inserts in Figure B1b) adjusting their positions to avoid overlap with $V$ s of other FDSs. The condition prohibiting $V$ overlap restricts total number of $V \mathrm{~s}$ and was used to check the values of $v_{n}$ obtained from the fit. The arrows of the circles contour line show the $V$ direction which is clockwise for $V \mathrm{~s}$ and anticlockwise for and $\mathrm{Vs} / \mathrm{aVs}$ and are color coded as is shown in the inserts in Figures 4a4c.

\section{Appendix D. Zero-field Majorana qubit}

In Fig. D1 we present the observation of the mixed $3(\infty)-9 / 7(\infty) \mathrm{em}^{\mathrm{M}}-\mathrm{C}$ states in the magneto-PL spectra of dot D07m, which correspond to the spectra measured at time delays $<4$ minutes, before equilibrium $3(\infty)$ state will be stabilized. The mixed states have

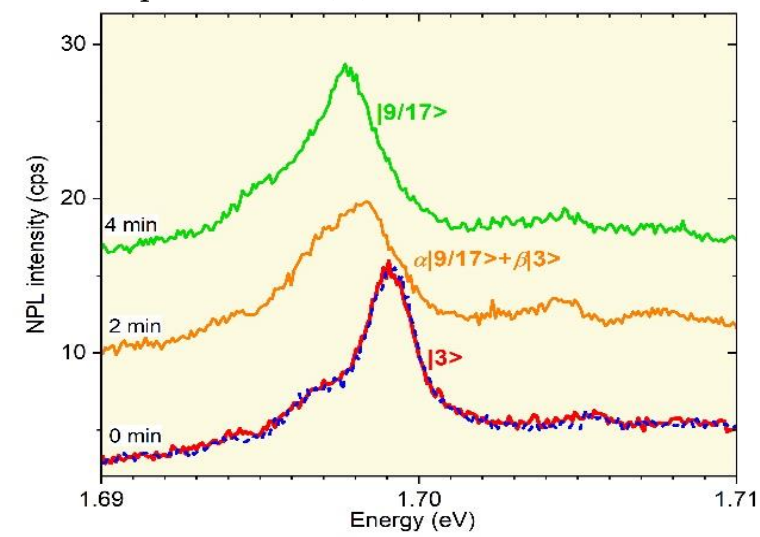

Figure D1. Spectra of D07m QD measured at $B \downarrow \downarrow=5 \mathrm{~T}$ at time delay 0, 2 and 4 minutes (solid curves) together with the spectra measured at $B_{e} \uparrow=5 \mathrm{~T}$ (dotted curve).

position of the s-peak between 0 (3) and 1 (19/7) qubit states as is seen in Figure D1 for delay $2 \mathrm{~min}$. 


\section{References}

1. Onnes H. K. Investigations into the Properties of Substances at Low Temperatures, Which Have Led, Amongst Other Things, to the Preparation of Liquid Helium. Nobel Lectures, 1913.

2. File J. and Mills R. G. Observation of persistent current in a superconducting solenoid. Phys. Rev. Lett. 1963, 10, 93-96.

3. Meissner W .and Oschenfeld R., Ein neuer effekt bei entritt der upraleitfähigkeit. Naturwissenschaften, 1933, 21, 787-788.

4. London F. Superfluids, John Willey \& Sons, New York, 1950, p. 152.

5. Deaver B. S. and Fairbank W. R. Experimental evidence for quantized flux in superconducting cylinders. Phys. Rev. Lett. 1961, 7, 43-46.

6. R. Doll and M. Näbauer, Experimental proof of magnetic flux quantization in a superconducting ring. Phys. Rev. Lett. 1961, 7, 51-52.

7. Abrikosov A. A. Type II superconductors and the vortex lattice. Nobel Lecture 2003.

8. Essmann U. and Träuble H. The direct observation of individual flux lines in type-II superconductors. Phys. Lett. A 1967, 24A, 526-527.

9. Cooper L. N. Microscopic quantum interference effects in the theory of superconductivity. Nobel Lecture 1972.

10. v Klitzing A K.; Dorda G.; Pepper M. New Method for High-Accuracy Determination of the Fine-Structure Constant Based on Quantized Hall Resistance. Phys. Rev. Lett. 1980, 45, 494-497.

11. Tsui D. C.; Stormer H. L.; Gossard A. C. Two-Dimensional Magnetotransport in the Extreme Quantum Limit, Phys. Rev. Lett. 1982, 48, 1559-1562.

12. Halperin B. I. Quantized Hall conductance, current carrying edge states and the existence of extended states in a two-dimensional disorder potential. Phys. Rev. B. 1982, 25, 2185-2190.

13. Jain J. K. Composite-fermion approach for the fractional quantum Hall effect. Phys. Rev. Lett 1989, 63, $199-202$.

14. Laughlin R. B. Anomalous Quantum Hall Effect: An Incompressible Quantum Fluid with Fractionally Charged Excitations. Phys. Rev. Lett., 1983, 50, 1395-1398.

15. Mintairov A. M.; Lebedev D.; Vlasov A.; Bogdanov A.; Ramezanpour S. and Blundell S. Fractional charge states in the magnetophotoluminescence spectra of a single-electron quantum dot, Nanomaterials, 2021, 11, 493-14.

16. Mintairov A. M.; Lebedev D. V.; Vlasov A. S.; Orlov A. O.; Snider G. L. and Blundell S. A. Nano-photoluminescence of natural anyon molecules and topological quantum computation. Sci. Rep. 2021, 11, 21440-11.

17. de Gennes P. G. Superconductivity of Metals and Alloys. Benjamin, New York, 1966, p. 294.

18. Chibotaru C. F.; Ceulemans A.; Bruyndoncx V.; Moshchalkov V. V. Symmetry induced formation of antvortexes in mesoscopic superconductors. Nature, 2000, 408, 833-835.

19. Slachmuylders A. F.; Partoens B.; and Peeters F. M. Geometry and magnetic-field-induced vortices and antivortices in mesoscopic two-dimensional systems. Phys. Rev. B 2005, 71, 245405-10.

20. Kitaev A. Fault-tolerant quantum computation by anyons, Ann. Phys. 2003 303, 2-20.

21. Millikan R. A. The Electron and the Light-Quant from the Experimental Point of View, Nobel Lecture , 1924.

22. Holton G. Subelectrons, Presuppositions, and the Millikan-Ehrenhaft Dispute, Historical Studies in the Physical Sciences , 1978, 9 161-224.

23. LaRue G. S.; Fairbank W. M. and. Hebard A. F. Evidence on the existence of fractional charge of matter. Phys. Rev. Lett. 1977, 38, 1011-1014.

24. Choi H. C.; Kang W.; Das Sarma S.; Pfeiffer L. N. \& West, K. W. Activation gaps of fractional quantum Hall effect in the second Landau level. Phys. Rev. B 2008, 77, 081301-4(R).

25. Willett R. L.; Nayak C.; Shtenge K.; Pfeiffer L. N. \& K.W. West, Magnetic-Field-Tuned Aharonov-Bohm Oscillations and Evidence for Non-Abelian Anyons at v =5/2. Phys. Rev. Lett. 2013, 111, 186401-5.

26. Volovik G. E. Fermion zero modes on vortices in chiral superconductors. JETP Lett. 1999, 70, 609-614.

27. Volovik G. E. Monopoles and fractional vortexes in chiral superconductors, PNAS, 2000, 97, 2431-2435.

28. Read N. and Green D. Paired states of fermions in two dimensions with breaking of parity and time-reversal symmetries and the fractional quantum Hall effect, Phys. Rev. B 2001, B 61, 10267-10297.

29. Ivanov D. A. Non-Abelian Statistics of Half-Quantum Vortices in p-Wave Superconductors. Phys. Rev. Lett. 2001, 86, 268-271.

30. Sarma S. D.; Freedman M. \& Nayak C. Majorana zero modes and topological quantum computation. npj Quantum Information 2015, 1, 15001-13.

31. Sarma S. D.; Freedman M.; Nayak C. Topologically Protected Qubits from a Possible Non-Abelian Fractional Quantum Hall State. Phys. Rev. Lett. 2005, 94, 166802-4.

32. Willett R. L.; Pfeiffer L. N.; West K. W., Alternation and interchange of e/4 and e/2 period interference oscillations consistent with filling factor 5/2 non-Abelian quasiparticles. Phys. Rev. B, 2010, 82, 205301-12.

33. Mintairov A. M.; Merz J. L.; Blundell S. Molecular States of Electrons: Emission of Single Molecules in Self-Organized InP/GaInP Quantum Dots, Fingerprints in the Optical and Transport Properties of Quantum Dots (InTech, 2012), pp. 125-152.

34. Mintairov A. M. et al. Control of Wigner localization and electron cavity effects in near-field emission spectra of In(Ga)P/GaInP quantum-dot structures. Phys. Rev B, 2018, 97, 195443-9.

35. Mintairov A. M. et al, Atomic ordering and bond relaxation in optical spectra of self-organized InP/GaInP2 Wigner molecule structures, Appl. Phys. Lett. 2019, 115, 202104-5. 
36. Mintairov A. M. et al. Piezo-electric fields and state-filling photo-luminescence in natural InP/GaInP2 Wigner molecule structures, Appl. Phys. Lett. 2021, 118, 121101-6.

37. Saarikoski H.; Harju A.; Puska M. J. and Niemin R. M. Vortex Clusters in Quantum Dots. Phys. Rev. Lett. 2004, 93, 116802-4.

38. Tavernier M. B.; E. Anisimovas and Peeters F. M. Ground state and vortex structure of the $\mathrm{N}=5$ and $\mathrm{N}=6$ electron quantum dot. Phys. Rev. B, 2006, 74, 125305-

39. Mourik V.; Zuo K.; Frolov S. M.; Plissard S. R.; Bakkers E. P. A. M.; Kouwenhoven L. P. Signatures of Majorana Fermions in Hybrid Superconductor-Semiconductor Nanowire Devices. Science, 2012, 336, 1003-1007.

40. Nadj-Perge S.; Drozdov I. K.; Li J.; Chen H.; Jeon S.; Seo J.; MacDonald A. H.; Bernevig B. A.; Yazdani A. Observation of Majorana fermions in ferromagnetic atomic chains on a superconductor. Science, 2014, 346, 602-607.

41. Xu J.-P. et al Experimental Detection of a Majorana Mode in the core of a Magnetic Vortex inside. Phys. Rev. Lett. 2015, 114, 017001-5.

42. Vinnikov L. Ya. et al Direct Evidence of Spontaneous Abrikosov Vortex State in Ferromagnetic Superconductor EuFe2(As1-xPx)2 with x = 0.21, arXiv:1709.09802 (2017).

43. Golod T.; Iovan A. \& Krasnov V. M., Single Abrikosov vortices as quantized information bits, Nat. Comm. 2015, 6 8628-5

44. Mintairov A. M.; He Y.; Merz J. L.; Jin Y.; Goldman R. S.; Kudrawiec R.; Misiewicz J.; Akimov I. A.; Yakovlev D. R.; Bayer M. Quasi-ordering of composition fluctuations and their interaction with lattice imperfections in an optical spectra of dilute nitride alloys, Semicond. Sci. Technol. 2016, 31, 095012-10.

45. Zhitenev N. B.; Fulton T. A.; Yacoby A.; Hess H. F.; Pfeiffer L. N.; West K. W. Imaging of localized electronic states in the quantum Hall regime. Nature, 2000, 404, 473-476.

46. Poux A.; Wasilewski Z. R; Friedland K. J.; Hey R.; Ploog K. H.; Airey R.; Plochocka P. and Maude D. K. Microscopic model for the magnetic-field-driven breakdown of the dissipationless state in the integer and fractional quantum Hall effect, $\underline{P h y s . R e v . ~} B_{,}$ 2016, 94, 075411-13

47. Gul Y. S.; Holmes N.; Myronov M.; Kumar S. and Pepper M. Self-organized fractional quantization in a hole quantum wire. J. Phys. Cond. Matter 2018, 30, 09LT01-6.

48. Kumar S.; Pepper M.; Holmes S. N.; Montagu H.; Gul Y.; Ritchie D. A. and Farrer I. Zero-Magnetic Field Fractional Quantum States. Phys. Rev. Lett. 2019, 122, 086803-5.

49. Ihnatsenka S. and Zozoulenko I. V. Origin of the " 0.25 anomaly" in the nonlinear conductance of a quantum point contact. $\underline{P h y s}$. Rev. B 2009, 79, 235313-5.

50. Czapkiewicz M.; Kolkovsky V.; Nowicki P.; Wiater M.; Wojciechowski T.; Wojtowicz T. and Wróbel J. Evidence for charging effects in CdTe/CdMgTe quantum point contacts. Phys. Rev. B 2012, 86, 165415-6.

51. Ilani S.; Martin J.; Teitelbaum E.; Smet J. H.; Mahalu D.; Umansky V. \& Yacoby A. The microscopic nature of localization in the quantum Hall effect, Nature, 2004, 427, 328-332.

52. Moore G. \& Read N. Nonabelions in the fractional quantum Hall effect. Nuclear Physics B 1991, 360, 362-396.

53. Greiter M.; Wen X. G. and Wilczek F. Paired Hall State at Half Filling, Phys. Rev. Lett. 1991, 66, 3205-3208.

54. J. Martin, S. Ilani, B. Verdene, J. Smet, V. Umansky, D. Mahalu, D. Schuh, G. Abstreiter, A. Yacoby, Localization of Fractionally Charged Quasi-Particles, Science, 2004, 305, 980-983.

55. Wojs A. and Hawrylak P. Theory of photoluminescence from modulation-doped self-assembled quantum dots Phys. Rev. B 1997, 55, 13066-1307.

56. Kyriakidis J.; Pioro-Ladriere M.; Ciorga M.; Sachrajda A. S. and Hawrylak P. Voltage-tunable singlet-triplet transition in lateral quantum dots, Phys. Rev. B. 2002, 66, 035320-8.

57. Warburton R. J.; Miller B. T.; Dürr C. S.; Bödefeld C.; Karrai K.; Kotthaus J. P.; Medeiros-Ribeiro G.; Petroff P. M.; and Huant S. Coulomb interactions in small charge-tunable quantum dots: A simple model. Phys. Rev. B, 1998, 58, 16221-231.

58. Wojs A. and Hawrylak P. Negatively charged magnetoexcitons in quantum dots, Phys. Rev. B. 1995, 51, 10880-10885.

59. Ediger M.; Bester G.; Badolato A.; Petroff P. M.; Karrai K.; Zunger A. and Warburton R. J. Peculiar many-body effects revealed in the spectroscopy of highly charged quantum dots. Nature Phys. 2007, 3, 774-779.

60. Jacak L.; Hawrylak P.; A. Wojs. Quantum Dots (Springer, Berlin, 1998), p. 176.

61. Yannouleas C. and Landman U. Unified microscopic approach to the interplay of pinned-Wigner-solid and liquid behavior of the lowest Landau-level states in the neighborhood of $v=1 / 3$. Phys. Rev. B 2011, 84, 165327-17.

62. Saint Jean M.; Even C. and Guthmann C. Macroscopic 2D Wigner islands, Europhys. Lett. 2001, 55, 45-51.

63. Stormer H. L.; Chang A.; Tsui D. C.; Hwang J. C. M.; Gossard A. C. and Weigmann W. Fractional quantization of the Hall effect, Phys. Rev. Lett. 1983, 50, 1953-1956. 